\title{
Chloroplast photosystem I dimer and high resolution model of the complex with plastocyanin
}

Andreas Naschberger ${ }^{1,2, \dagger}$, Laura Mosebach $^{3, \dagger}$, Victor Tobiasson ${ }^{1,2}$, Sebastian Kuhlgert ${ }^{3}$, Martin

6 Scholz $^{3}$, Annemarie Perez-Boerema ${ }^{1}$, Yuichiro Takahashi ${ }^{4,5}$, Michael Hippler ${ }^{3,6^{*}}$, Alexey 7 Amunts ${ }^{1,2, *}$

${ }^{1}$ Science for Life Laboratory, Department of Biochemistry and Biophysics, Stockholm 10 University, 17165 Solna, Sweden

$11{ }^{2}$ Department of Medical Biochemistry and Biophysics, Karolinska Institute, 17177 Stockholm,

12 Sweden

13 Institute of Plant Biology and Biotechnology, University of Münster, 48143 Münster, Germany

$14{ }^{4}$ Research Institute for Interdisciplinary Science, Okayama University, Okayama 700-8530, Japan

15 Japan Science and Technology Agency-CREST, 4-1-8 Kawaguchi, Saitama 332-0012, Japan

$16{ }^{6}$ Institute of Plant Science and Resources, Okayama University, Kurashiki, Japan

*Correspondence to: mhippler@uni-muenster.de; amunts@scilifelab.se

$\dagger$ These authors contributed equally to this work.

\section{Abstract}

24 Photosystem I (PSI) enables photo-electron transfer and regulates photosynthesis in the bioenergetic membranes of cyanobacteria and chloroplasts. Being a multi-subunit complex, its macromolecular organization affects the dynamics of photosynthetic membranes. Here, we reveal a chloroplast PSI from the green alga Chlamydomonas reinhardtii that is organized as a homodimer, comprising 40 protein subunits with 118 transmembrane helices that provide scaffold for 568 pigments. Our cryo-EM structure identifies the light-harvesting protein Lhca9 as the key element for the dimerization. Furthermore, the absence of Lhca2 and PsaH, gives rise to a head-to-head relative orientation of the PSI-LHCI monomers, in a way that is essentially different from the oligomer formation in cyanobacteria. The interface between the monomers partially

33 overlaps with the surface area that would bind one of the LHCII complexes in state transitions.

34 We also define the most accurate available PSI-LHCI model at $2.3 \AA$ resolution, including a 35 flexibly bound electron donor plastocyanin, and assign correct identities and orientations of all the pigments, as well as 486 water molecules that affect energy transfer pathways. 
Main

A chloroplast PSI of green algae consists of the core complex and three antenna modules: inner belt, outer belt, and Lhca2:Lhca9 heterodimer, which together comprise 24 subunits ${ }^{1-4}$. As a short-term light acclimation mechanism in response to a fluctuating illumination and anoxia, the algal PSI additionally associates with two LHCII trimers ${ }^{5,6}$. Structural studies have shown that the oligomeric state of a chloroplast PSI is a monomer, due to a presence of the subunit PsaH, whereas in cyanobacteria also structures of dimers ${ }^{7-9}$ and trimers 10 were reported. Cyanobacterial PSI oligomerizes via direct contacts between subunits PsaI and PsaL, however such an association has been ruled out for a chloroplast PSI due to structural constraints and apparent rigidity ${ }^{11,12}$. Recent structural studies of PSI from a chloroplast of a salt-tolerant alga suggest that its functional core may vary more than previously believed, thus suggesting a potential architectural plasticity in response to environment ${ }^{13}$. On the macromolecular level, an atomic force microscopy analysis of a plant thylakoid membrane showed that when its architecture is altered upon transition from darkness to light, larger inter-membrane contacts are formed, leading to a reduced diffusion distance for the mobile electron carriers ${ }^{14}$. The membrane architecture in dark- and light-adapted membranes contains ordered rows of closely packed PSI dimers, which are more abundant in the dark state ${ }^{14}$. Similarly, closely associated PSI-LHCI complexes were detected in plants by negative stain electron microscopy ${ }^{15}$. This suggests that reversible PSI dimer formation may have a physiological role in thylakoid membrane structure maintenance in chloroplasts. However, very little is known about chloroplast PSI-LHCI dimers, and information on their structures is lacking. In the absence of any structural data, no evidence is available on composition, elements mediating dimerization, and how the arrangement would differ from the cyanobacterial counterparts.

To study a chloroplast PSI dimer at high resolution, we grew genetically modified C. reinhardtii cells containing a His-tag at the N-terminus of PsaB in low light and under anoxic conditions (see Methods). The thylakoid membranes were then solubilised with $n$-dodecyl- $\alpha-D$-maltoside ( $\alpha$ DDM), followed by affinity purification, crosslinking via the chemically activated electron donor plastocyanin (Pc) and sucrose density gradient centrifugation (Extended Data Fig. 1). Two PSI fractions were detected on the sucrose gradient. 2D polyacrylamide gel electrophoresis (native/reducing 2D-PAGE) of isolated thylakoids indicated the presence of PSI dimers (Extended Data Fig. 2 and Supplementary Table 1). The heavier green band on the gradient was subjected to single-particle cryo-EM analysis (Supplementary Table 2). We used 2D classification to separate PSI dimers from monomers in a reference free manner, followed by $3 \mathrm{D}$ classification leading to a subset of 14,173 particles, which were refined to an overall resolution of $2.97 \AA$ by applying C2 symmetry (Extended Data Fig. 3). Upon symmetry expansion, the resolution was further improved to $2.74 \AA$ (Extended Data Fig. 3). The remaining 74,209 particles containing the monomer were refined to $2.31 \AA$ resolution, representing a considerable improvement on the previously reported 2.9-3.5 $\AA$ resolution structures ${ }^{1-3}$, as well as X-ray crystal structures of a plant PSI ${ }^{16,17}$. A density corresponding to the bound electron donor Pc was found at the luminal side of both PSI forms, dimer and monomer. 
78 To derive a structure of the chloroplast PSI dimer, we first built an accurate model of one

79 monomer using the $2.74 \AA$ resolution map, and then fitted it into the cryo-EM density of the C2

80 refined dimer. Compared to the monomer, all but two core subunits (PsaH, PsaO) and one light-

81 harvesting protein (Lhca2) are found in the dimer (Fig. 1). The structure contains 40 protein

82 subunits, 398 chlorophylls $a, 60$ chlorophylls $b, 56$ beta-carotenes, 54 luteins, 2 violaxanthins, 2

83 neoxanthins, 4 phylloquinones, 6 iron-sulphur clusters, and 32 lipids (Fig. 1). In addition, two

84 unaccounted densities corresponding to two loops in the stromal side of Lhca9 and PsaG could be

85 interpreted in the dimer, due to the stabilization by the adjacent monomer (Extended Data Fig. 4).

86 The first better-defined density is Lhca9 loop region 132-153 which is stabilised due to a direct

87 interaction with PsaL of the second monomer within the dimer. As a result, the area is closely

88 packed with PsaG, and therefore also the PsaG loop region 63-77 is better resolved in the dimer

89 (Extended Data Fig. 4). Finally, cofactors of Lhca9 could be modelled at the interface between

90 the monomers. No density for the His-tag on PsaB could be detected. 
bioRxiv preprint doi: https://doi.org/10.1101/2021.08 30.458224; this version posted August 31,2021. The copyright holder for this preprint (which was not certified by peer review) is the author/funder, who has granted bioRxiv a license to display the preprint in perpetuity. It is made available under aCC-BY 4.0 International license.
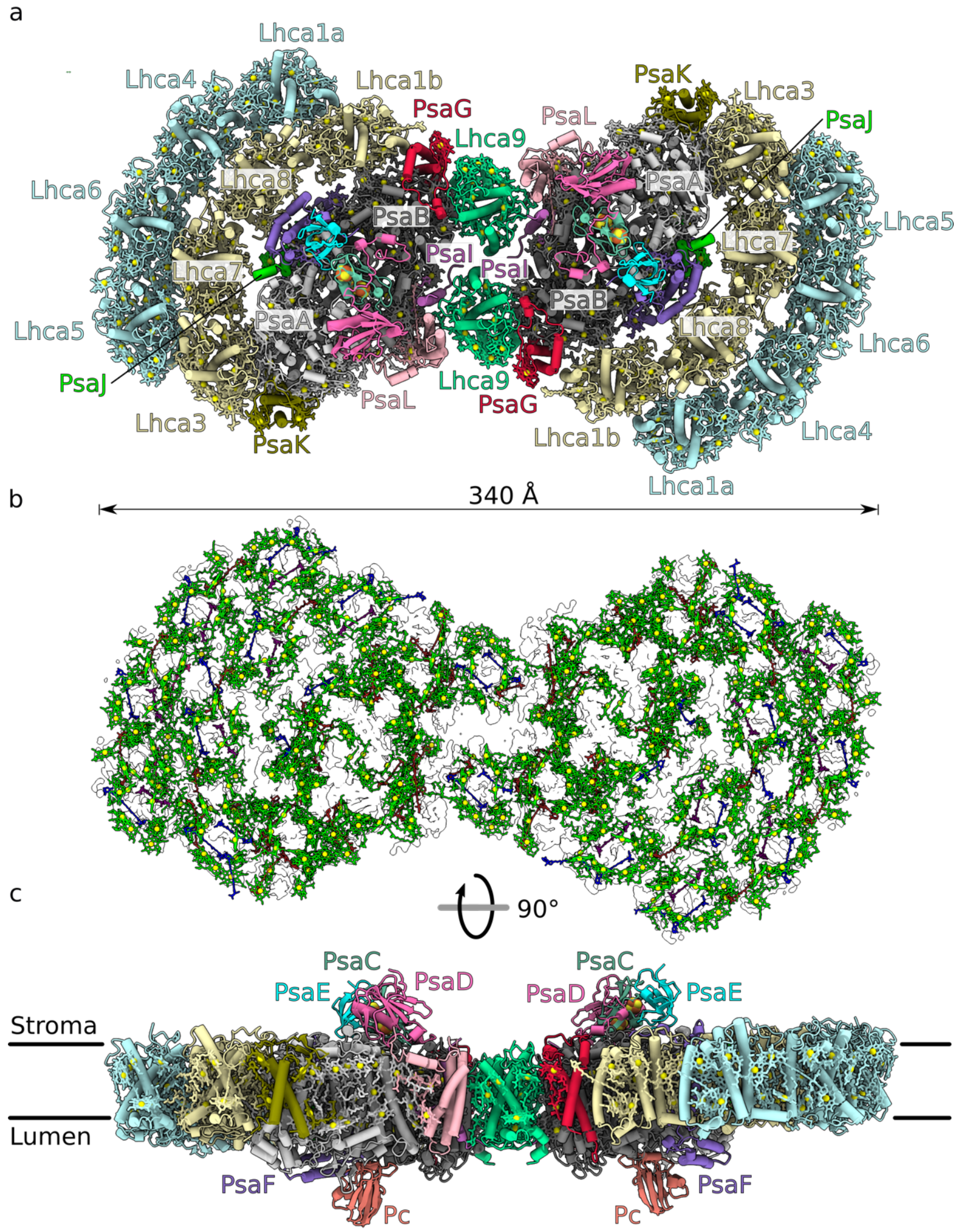

92 Fig. 1: Overall structure of the PSI dimer. a, View of individual proteins from stroma. b,

93 Arrangement of the pigments in the outline of the map: chlorophylls green (Mg yellow), luteins

94 blue, beta-carotenes red, violaxanthin purple, and neoxanthin pink c, Overall view along the

95 membrane. 
The structural basis for the algal chloroplast PSI dimerization is fundamentally different from cyanobacteria (Fig. 2a). In cyanobacteria, PSI dimerises via the stromal region of PsaL ${ }^{7-9}$ and trimerises via the lumenal C-terminus of PsaL, assisted by PsaI ${ }^{10}$. In our structure of the chloroplast PSI-LHCI dimer, neither PsaL nor PsaI interact with each other between the neighbouring units. Instead, PsaH that preserved a monomer is removed, and Lhca9 and its associated cofactors act as a symmetrical linker between the monomers, highlighting the importance of the light-harvesting antenna proteins for regulation of the macro-organisation. Lhca9 is distinct among the light-harvesting proteins due to a truncated loop between helices A and $\mathrm{C}$, and lack of the associated chlorophyll ${ }^{6}$. Based on this difference, we rationalised how Lhca9 allows for dimerization, as a longer AC-loop would clash with the neighbouring PsaB (Extended Data Fig. 5), although conformational changes cannot be excluded.

107 Consequently, the two Lhca9 copies tether the PSI monomers in a head-to-head fashion, resulting 108 in a 340- $\AA$ long structure (Fig. 1 and Fig. 2b). They form interactions of four types covering the entire membrane span: 1) a hydrogen bond of the backbone carbonyl of G148 with S137 of PsaL in the stroma; 2) a hydrogen bond between the two Q109 of the Lhca9 copies; 3) hydrophobic contacts via coordinated cofactors in the membrane that include a newly modelled, beta-carotene 623, and five chlorophylls $(604,610,611,612,810)$; 4) lipid-mediated hydrophobic interactions

113 via monogalactosyl diglyceride LMG852 and LMU624. One acyl chain of lipid 852 associates 114 with chlorophyll 810 from monomer-2, while the other acyl chain associates with beta-carotene 115 BCR623 from monomer-1 (Fig. 2b). Thus, lipids contribute to the oligomerization of PSI, 116 meaning that the membrane itself plays a role in the association. The finding that specific 117 carotenes and lipids enable inter-molecular contacts that bridge the PSI monomers is of a 118 particular interest, as it can only be detected by high-resolution structural studies. The 119 involvement of lipids in PSI oligomerization is consistent with the formation of supercomplexes 120 in other bioenergetic membranes ${ }^{18-21}$.

121 To solidify the structural observations, we engineered a $\Delta l h c a 9$ insertional mutant having the His122 tag at the N-terminus of PsaB and repeated the purification procedure in the same way as for the 123 wild type. This time, no PSI dimer band could be found in the sucrose density gradient (Extended 124 Data Fig. 6). Next, we analysed sucrose density gradients of solubilized thylakoids from $\Delta$ lhca 2 125 and $\Delta$ lhca 9 mutants without the His-tag (Extended Data Fig. 7). Compared to the wild type, in 126 Alhca2, the abundance of the heavier band was reduced, and Lhca9 is present, supporting its 127 independent association with PSI. However, in $\Delta l$ lhca 9 , no PSI dimer fraction was observed, and 128 Lhca2 presence is reduced. This data is consistent with the proposed structural role of Lhca9 in 129 the PSI dimer formation. 

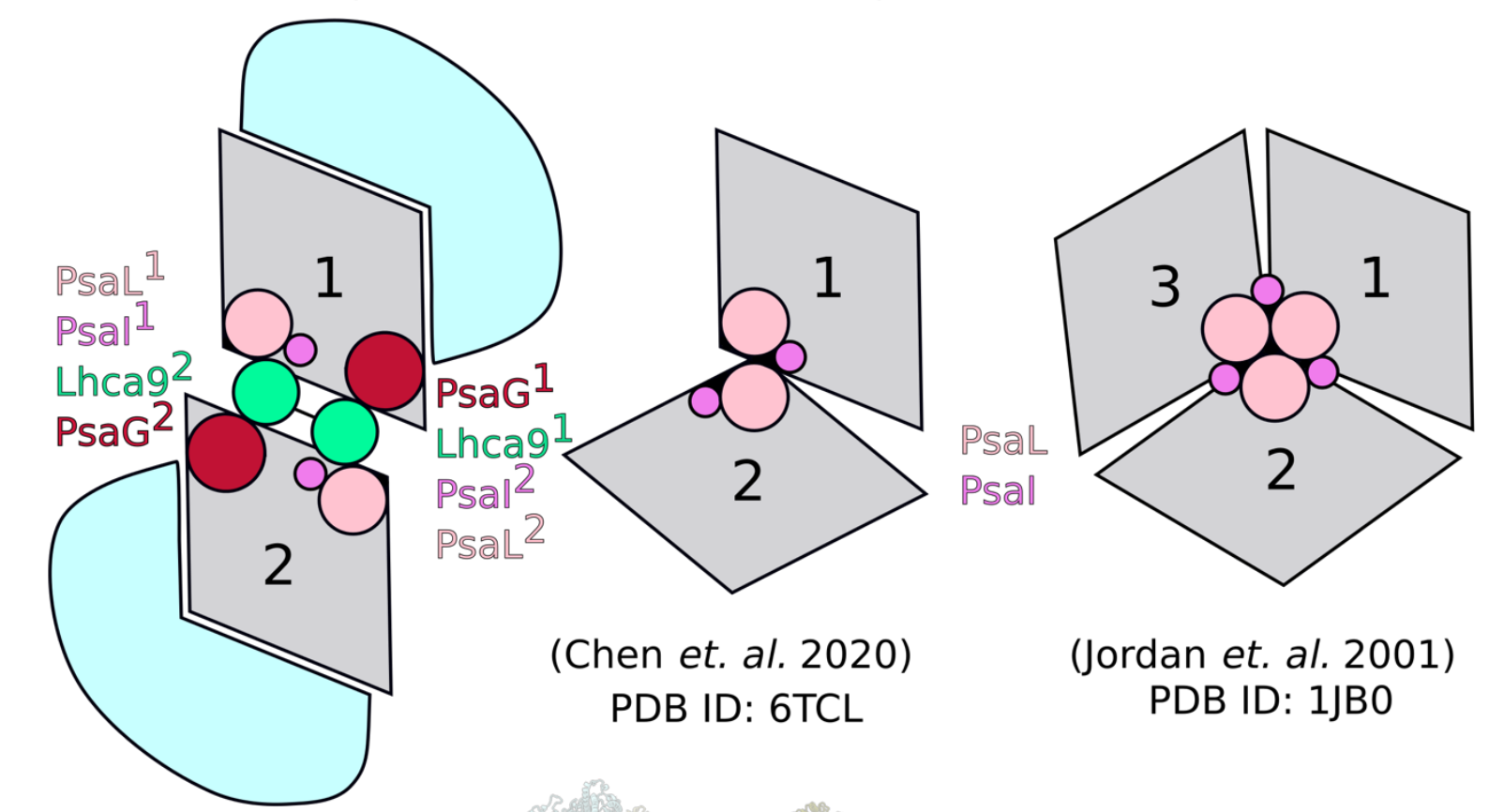

(Jordan et. al. 2001) PDB ID: 1 JB0

b

(Chen et. al. 2020)

PDB ID: 6TCL

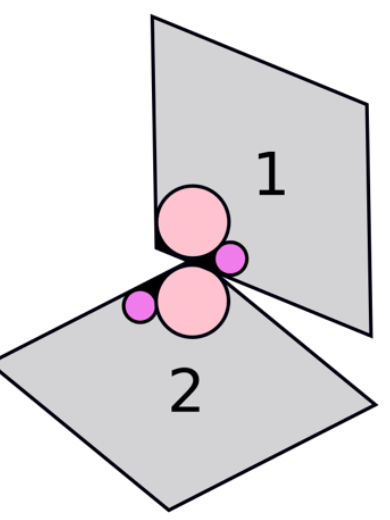

Fig. 2: Dimerization of PSI. a, Schematic representation: Chloroplast PSI-LHCI dimer associated via Lhca9, cyanobacterial PSI dimer (PDB ID: 6TCL) and trimer (PDB ID: 1JB0) associated via PsaI/L. The PSI core is grey, LHCI light-blue. b, The dimer interface is formed by: hydrogen bonds between PsaL and Lhca9, and between two Lhca9 copies (left); potential energy transfer paths between the two monomers (center); pigments and lipids (right).

The specific interactions between the monomers are enabled due to unoccupied positions of Lhca2 and PsaH (Fig. 3a). Compared to the state transition complex 5,6, Lhca9 from the neighbouring monomer is positioned in the membrane, where Lhca2 resides in PSI-LHCI-LHCII (Fig. 3b,c), and their three transmembrane helices would overlap with each other (Fig. 3c). The presence of the PsaH transmembrane helix is not compatible with the Lhca92-associated cofactors CLA9, LMG852, BCR9 that extend from the neighbouring monomer in the dimer (Fig. 3c). In 
142 addition, the superposition shows that there would be a clash between PsaG and Lhcal of the 143 inner belt with LHCII-2 (Fig. 3c). Since Lhca2 and PsaH are required for the lateral binding of 144 LHCII to the core in state transitions, the structure of the algal PSI dimer would not facilitate 145 LHCII binding at this position. However, our 2D-PAGE indicated a comigration of LHCII 146 polypeptides with the dimer fraction, and therefore a structural adaptation cannot be excluded. 147 The antagonistic relationship of Lhca $9^{2}$ and Lhca2 might reflect a regulation of PSI dimerization 148 that is associated with the membrane shape adaptation in response to environment ${ }^{14}$.

149 Compared to algae, plant PSI binds one LHCII trimer with a stronger association to the core, the 150 docking site of which is further away from the PsaL side ${ }^{22}$. Larger than monomer fractions of a 151 plant PSI have been reported ${ }^{23}$, and we do not observe a moiety from the dimer that would affect 152 the contact surface of a plant LHCII. Since 2D projections from negative stain images of 153 Arabidopsis PSI suggest the presence of a Lhca hetero-dimer that is analogous to alga ${ }^{24}$, a state 154 transition complex can be in principle structurally established with the PSI dimer in plants (Fig. $1553 \mathrm{~d})$.
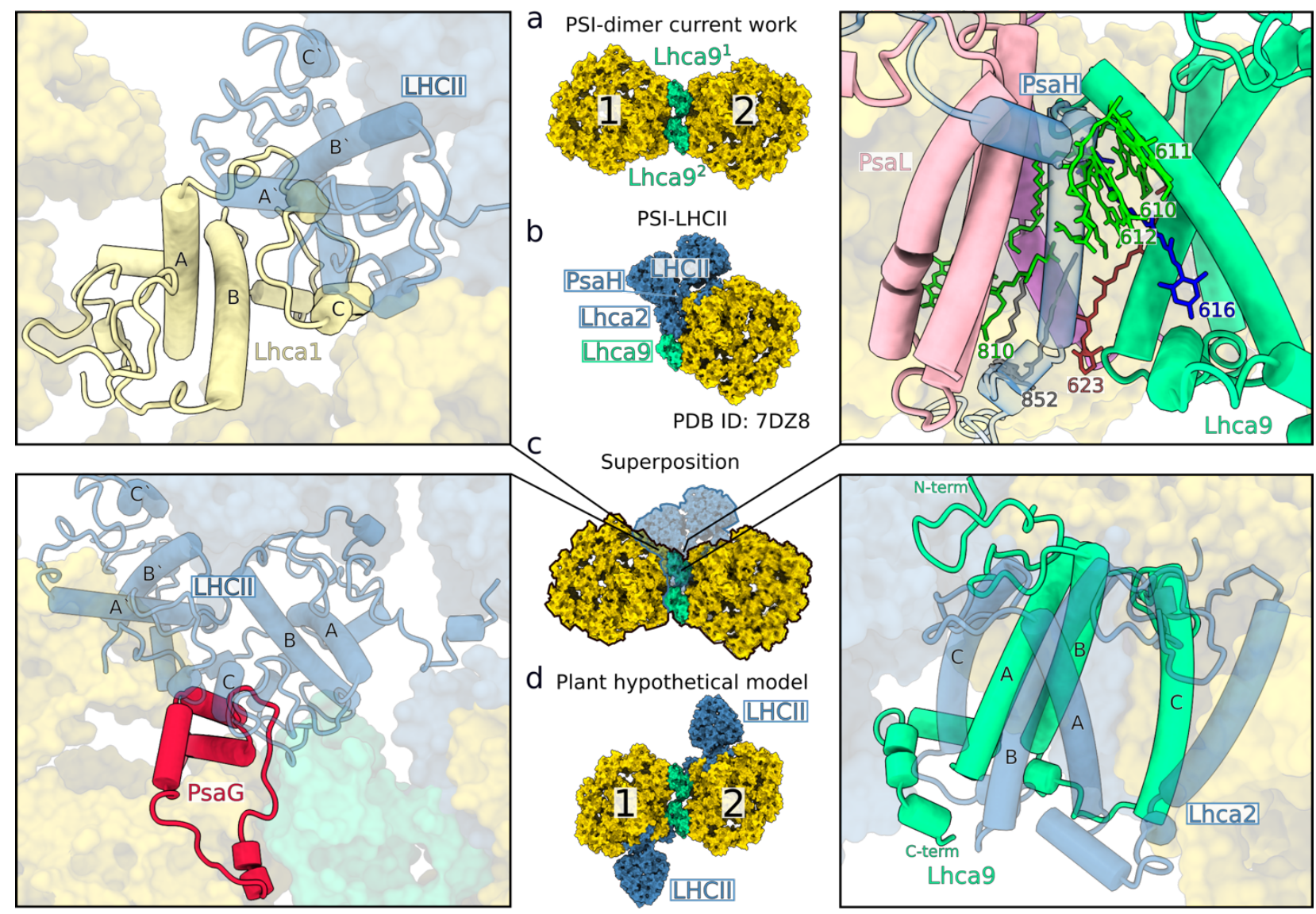

157 Fig. 3: PSI dimer and LHCII association in C. reinhardtii. a, The PSI dimer of the current work. b, The PSI with LHCII in state transition (PDB ID: 7DZ8). c, Superposition of PSI dimer with PSI-LHCII shows clashes of Lhca2:LHCII with PsaG, PsaL, Lhca1, Lhca9 from the neighbouring PSI monomer. d, A hypothetical model of a plant PSI dimer with a single LHCII 161 copy is presented. 
162 To further extrapolate potential conformational changes during the dimerization of PSI, we 163 applied multi-body refinement analysis of the PSI dimer, using the two monomers as bodies 164 (Extended Data Fig. 8). The analysis indicated no distinct conformational states, but instead 165 revealed continuous motions in the three eigenvectors describing a relative movement of the 166 monomers in relation to each other (Extended Data Fig 8a). The intrinsic flexibility is dominated 167 by combinations of all three rotations of one monomer with respect to the other up to $13^{\circ}$ 168 (Extended Data Fig. 8b-d). Therefore, excitation energy transfer between the PSI monomers in 169 the dimeric scaffold would also depend on degrees of rotation around the identified pivot points. 170 Specifically, three chlorophylls are found within a potential cross-monomer excitation-sharing: 171 CLA807 (PsaB), CLA604 (Lhca9²), CHL606 (Lhca9²), and the distance between them is $20 \AA$ 172 in the consensus map (Fig. 2). While such a positioning might suggest direct coupling, the multi173 body analysis indicates a considerable variability (Extended Data Fig. 8e, 8f). Therefore, similar 174 to the cyanobacterial PSI dimer, an excitation coupling between the two monomers is less 175 favourable in vitro, and this is consistent with measurements of $77 \mathrm{~K}$ fluorescence spectra that 176 showed only a minor shift between monomer and dimer (Extended Data Fig. 1). However, in vivo 177 the observed PSI-LHCI dimer conformation, and therefore the distance between the chlorophylls 178 at the interface, could also be affected by a local membrane curvature.

179 In our PSI monomer reconstruction, the resolution in the core is $\sim 2.1 \AA$, and in the LHCI inner 180 belt 2.1-2.5 $\AA$, revealing unprecedented structural details of chlorophylls, carotenoids, and water 181 molecules (Fig. 4, Extended Data Fig. 4 and 10, Supplementary Table 2). The map can improve 182 the level of detail not only when compared to the previous cryo-EM studies of algal PSI ${ }^{1-3}$, but 183 also the plant PSI maps obtained by X-ray crystallography ${ }^{16,17}$ (Extended Data Fig. 9). The 184 quality of the data aided in improving the previous models in functionally important regions. This 185 includes the identification of nine $\mathrm{Chl} b$ molecules, two newly modelled luteins, a beta-carotene 186 and more accurate estimation of the coordination of 53 chlorophylls (Fig. 4b, Extended Data Fig. 187 10). Particularly, Chl $b$ molecules are identified at positions 601 and 606 in Lhca4, Lhca5, Lhca6, 188 Lhca7 and Lhca8. The two newly modeled luteins 720 and 626 are in the N-terminus of Lhca3 189 next to Chl $a$ 614, and in Lhca5 next to Chl a 617, respectively (Fig. 4b). The newly modeled 190 beta-carotene 622 is in Lhca9 and could be identified due to structural stabilization of the 191 interface region in the dimer (Fig. 4b). Since chl $b$ limits free diffusion of excitation energy ${ }^{25}$, 192 some of the new assignments affect the energy pathways between the antenna proteins. Together 193 with the new structural data, this allowed us to produce a more accurate map of the energy 194 channelling in PSI based on the new model (Fig. 4a).

195 In addition, on the luminal side of PSI, we observed a density corresponding to the associated 196 electron donor Pc. Signal subtraction, followed by focused 3D classification, allowed us to rigid 197 body fit a model for Pc into the density at the local resolution of $\sim 3.5 \AA$ (Extended Data Fig. 3). 198 We then performed flexible fitting using self-similarity-restraints in Coot $^{26,27}$. With respect to the 199 PSI-Pc interactions, comparison between our model with a plant counterpart ${ }^{28,29}$ revealed two 200 main differences (Extended Data Fig. 11). In C. reinhardtii, the negatively charged residues of 201 the Pc acidic patch are shifted by $\sim 5$ due to the missing residues P58-E59, and therefore, the 202 interaction with K101 of PsaF is weakened (Extended Data Fig. 11). Instead, the binding strength 
203 is compensated with the PsaF region K78-K92, which has six lysines $(78,81,82,85,89,92)$

204 increasing a positively charged concentration at a distal site (Extended Data Fig 11c,d), thus

205 supporting additional electrostatic interactions with the acidic residues of Pc. The importance of

206 the distal lysine residues is supported by site-directed mutagenesis of the C. reinhardtii N-

207 terminal PsaF domain and functional analyses of electron transfer between Pc and mutant as well

208 as wild type PSI ${ }^{30}$. Thus, algae and plant Pc adapted their slightly different interfaces for optimal

209 interactions with PSI.

210 A striking feature of the high resolution cryo-EM map is resolvable density for multiple newly

211 detected water molecules, which particularly aided in modeling the coordination of chlorophylls

212 (Fig. 4c). Thus, we report the most complete available experimental picture of a chemical

213 environment for chlorophyll binding (Supplementary Table 3). Particularly, it allows

214 distinguishing between mono- and di-hydrated forms (Extended Data Fig. 12), which largely

215 escaped detection by X-ray crystallography (Extended Data Fig. 9). This is mechanistically

216 important, because the di-hydrated derivative is chemically more stable, as illustrated by quantum

217 chemical calculations ${ }^{31}$. We observe that, other than the previously reported CLA824 ${ }^{16}$, only

218 two waters can be involved in penta-coordinated $\mathrm{Mg}$ for all the chlorophylls. Remarkably, waters

219 play a coordinative role for most of $\mathrm{Chl} b$, for which the relative ratio of water coordination is

220 four times higher than for Chl $a$ (Supplementary Table 3). The difference between Chl $a$ and Chl

$221 b$ is a methyl versus a formyl group, thus water serves as a hydrogen bond donor to the latter,

222 while it also interacts with charged/polar protein residues or lipids. Therefore, the immediate

223 surrounding of $\mathrm{Chl} b$ molecules is more enriched with non-protein material than previously

224 thought, which plays a role in tuning the photophysics and the transport properties of excitation

225 energy in PSI. Together, the presented model allows now for comparison of PSI phylogenetic

226 conservation also on the level of chlorophyll coordination and solvent positioning. 


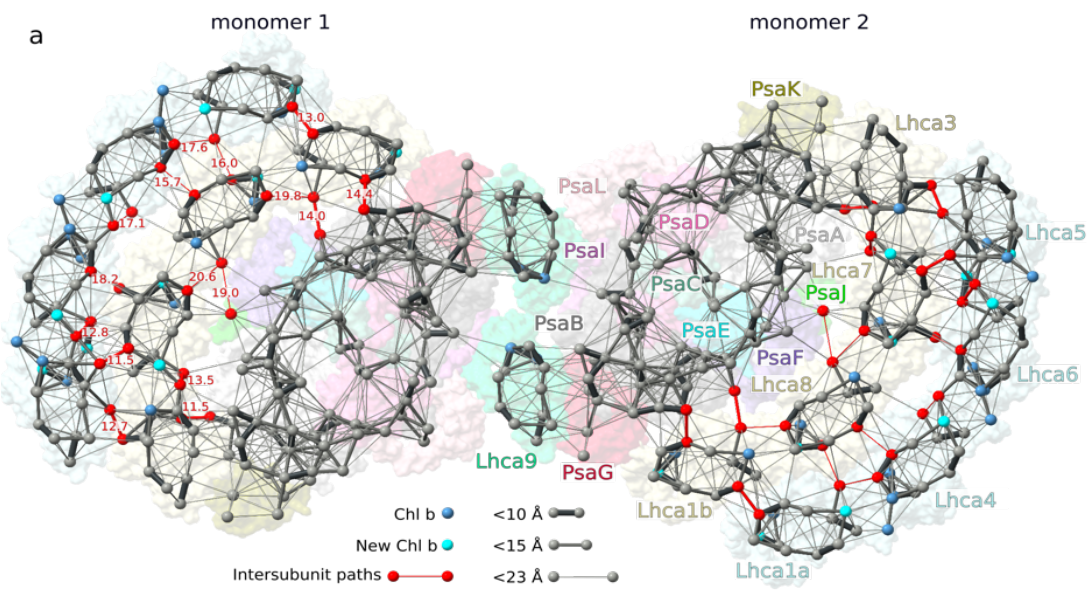

b
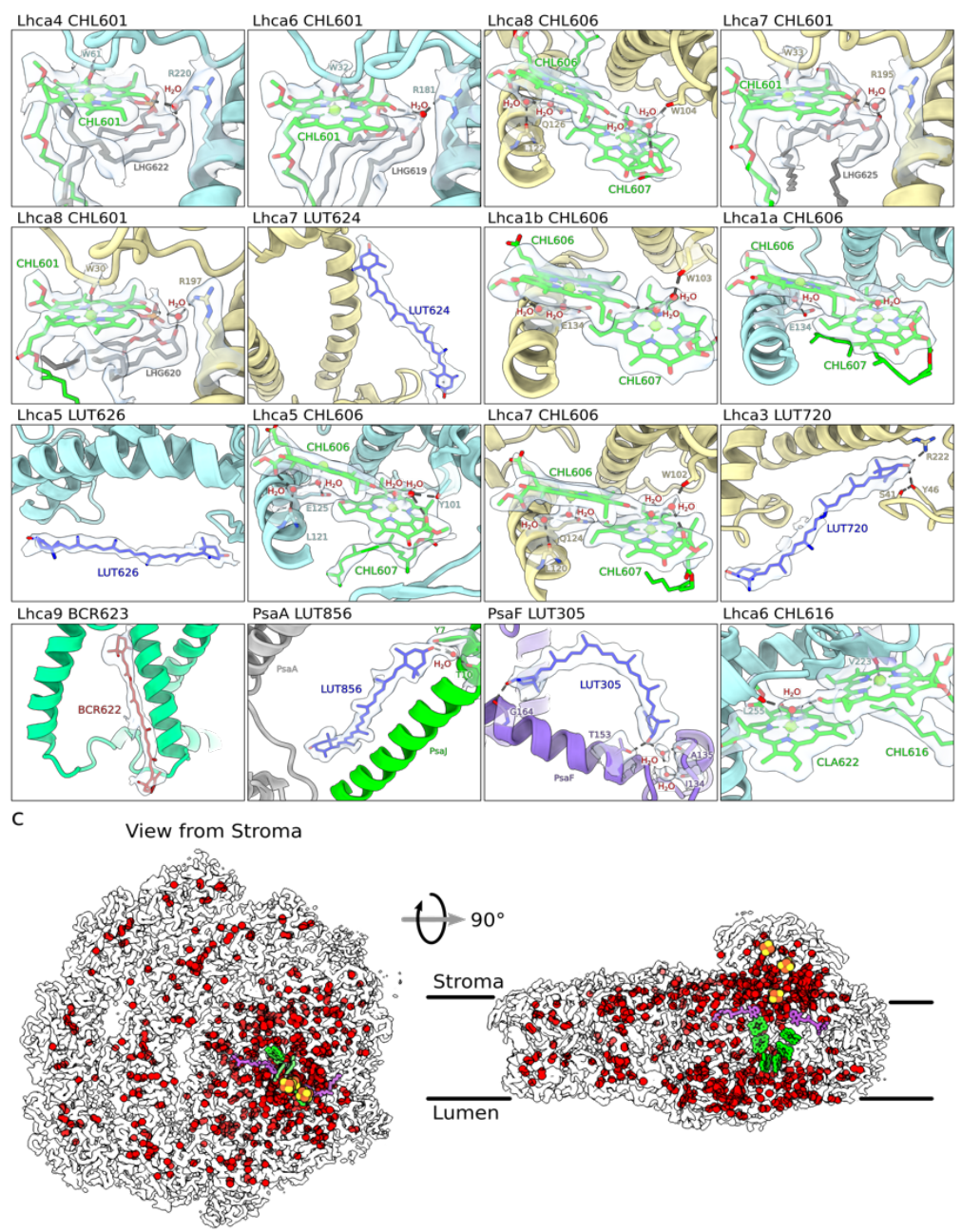

228 Fig. 4: High resolution features of co-factors and solvation. a, Energy transfer pathways. Distribution of chlorophylls indicated by $\mathrm{Mg}$ atoms. Chl $a$ gray, Chl $b$ blue, newly identified Chl $b$ cyan, pathways within $23 \AA$ are connected by lines, and the line width reflects the distance. The most likely intersubunit pathways are red. b. Close up views of newly identified elements, coordination and chemical environment with the density map. c, Water molecules modeled in PSI are shown as red spheres (oxygen atoms) in the outline of the map, chlorophyll green, phylloquinones purple, iron-sulfur clusters yellow-red. 


\section{Methods}

\section{Strains and growth conditions}

237 Experiments were performed a previously described strain expressing PsaB fused with His ${ }_{20}$-tag after the third residue from the N-terminus ${ }^{2}$ and an lhca 9 insertional mutant ${ }^{32}$ transformed with the corresponding PsaB His tag plasmid. Chloroplast transformation and transformant selection was performed as described previously. Further experiments were performed with the $C$. reinhardtii wild type strain $137 \mathrm{c}$ as well as $l$ hca 2 and $l$ hca 9 insertional mutant ${ }^{32}$ back-crossed into wild type 137c. presence of $\sim 20 \mu \mathrm{mol}$ photons $\mathrm{m}^{-2} \mathrm{~s}^{-1}$ photosynthetically active, continuous illumination. For experiments, strains were cultured in TAP medium on a rotary shaker $(120 \mathrm{rpm})$ at $25^{\circ} \mathrm{C}$ in the presence of $\sim 20 \mu \mathrm{mol}$ photons $\mathrm{m}^{-2} \mathrm{~s}^{-1}$ photosynthetically active, continuous illumination.

\section{Purification of PSI}

C. reinhardtii cells featuring were incubated in anoxic conditions $\left(\sim 10^{8}\right.$ cells $\mathrm{mL}^{-1}$ in TAP medium $+10 \mathrm{mM}$ glucose, $40 \mathrm{U} \mathrm{mL}^{-1}$ glucose oxidase, $50 \mathrm{U} \mathrm{mL}^{-1}$ catalase $)$ and dim light ( 20 $\mu \mathrm{mol}$ photons $\mathrm{m}^{-2} \mathrm{~s}^{-1}$ ) for $60 \mathrm{~min}$. All following steps were performed at $4{ }^{\circ} \mathrm{C}$ and $\operatorname{dim} \operatorname{light}$. Cells were disrupted in $0.33 \mathrm{M}$ sucrose, $25 \mathrm{mM}$ HEPES-KOH pH $=7.5,5 \mathrm{mM} \mathrm{MgCl} 2,1 \mathrm{mM}$ PMSF, 1 $\mathrm{mM}$ benzamidine, $5 \mathrm{mM}$ aminocaproic acid with a nebulizer ( 2 bar, two passages). Thylakoid membranes were extracted via sucrose density step gradient ultracentrifugation $(0.5 \mathrm{M}-1.3 \mathrm{M}-$ $1.8 \mathrm{M}$ sucrose, $5 \mathrm{mM}$ HEPES-KOH $\mathrm{pH}=7.5,10 \mathrm{mM}$ EDTA, $1 \mathrm{mM}$ benzamidine, $5 \mathrm{mM}$ aminocaproic acid). Isolated thylakoids were set to $1 \mathrm{mg} \mathrm{chl} \mathrm{mL}^{-1}$ in $5 \mathrm{mM}$ HEPES-KOH $\mathrm{pH}=7.5$ and solubilized by addition of an equal volume of $2 \% \alpha$-DDM for $10 \mathrm{~min}$. Unsolubilized material was separated by centrifugation. The supernatant was diluted four times to $125 \mu \mathrm{g} \mathrm{chl} \mathrm{mL} \mathrm{m}^{-1}$ and $0.25 \% \alpha$-DDM. The sample was loaded onto a TALON metal affinity column $\left(1 \mathrm{~mL}\right.$ resin $\left.\mathrm{mg} \mathrm{chl}^{-1}\right)$ in $5 \mathrm{mM}$ HEPES-KOH $\mathrm{pH}=7.5,100 \mathrm{mM} \mathrm{NaCl}, 5 \mathrm{mM} \mathrm{MgSO}_{4}, 10 \%$ glycerol at a flow rate of $\sim 0.5 \mathrm{~mL}$ $\mathrm{min}^{-1}$. The column was washed with 10 times the bed volume of $5 \mathrm{mM}$ HEPES-KOH $\mathrm{pH}=7.5$, $100 \mathrm{mM} \mathrm{NaCl}, 5 \mathrm{mM} \mathrm{MgSO}_{4}, 10 \%$ glycerol, $0.02 \% \alpha$-DDM at a flow rate of $\sim 1 \mathrm{~mL} \mathrm{~min}^{-1}$. A second wash was performed with 10 times the bed volume of $5 \mathrm{mM} \mathrm{HEPES-KOH} \mathrm{pH}=7.5,100$ $\mathrm{mM} \mathrm{NaCl}, 5 \mathrm{mM} \mathrm{MgSO}_{4}, 10 \%$ glycerol, $0.02 \% \alpha$-DDM and $5 \mathrm{mM}$ imidazole at a flow rate of $\sim 1 \mathrm{~mL} \mathrm{~min}{ }^{-1}$. The PSI was eluted with $5 \mathrm{mM}$ HEPES-KOH $\mathrm{pH}=7.5,100 \mathrm{mM} \mathrm{NaCl}, 5 \mathrm{mM}$ $\mathrm{MgSO}_{4}, 10 \%$ glycerol, $0.02 \% \alpha$-DDM and $150 \mathrm{mM}$ imidazole. The PSI was concentrated with a

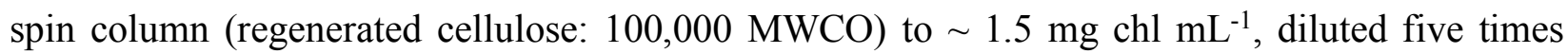
with $30 \mathrm{mM}$ HEPES-KOH pH $=7.5,0.02 \% \alpha$-DDM and reconcentrated twice. Pc crosslinking was performed in $30 \mathrm{mM}$ HEPES-KOH $\mathrm{pH}=7.5,1 \mathrm{mM}$ ascorbate, $0.1 \mathrm{mM}$

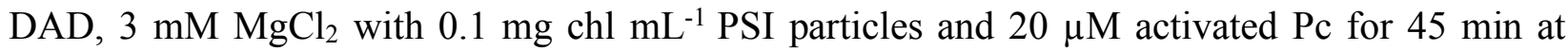
room temperature. Pc activation was performed in $10 \mathrm{mM}$ MOPS-KOH pH $=6.5$ with $100 \mu \mathrm{M}$ recombinant $\mathrm{PC}, 5 \mathrm{mM} \mathrm{EDC}$ and $10 \mathrm{mM}$ sulfo-NHS for $20 \mathrm{~min}$ at room temperature. The crosslinker was removed and the buffer exchanged to $30 \mathrm{mM}$ HEPES-KOH $\mathrm{pH}=7.5$ via a PD 
G25 desalting column followed by ultrafiltration with a centricon (regenerated cellulose: 10,000 MWCO). strains were loaded onto a $1.3 \mathrm{M}-0.1 \mathrm{M}$ sucrose density gradient including $5 \mathrm{mM}$ HEPES-KOH $\mathrm{pH}=7.5$ and $0.02 \% \alpha$-DDM. PSI fractions were collected after ultracentrifugation at 33,000 rpm (Beckman Coulter SW $41 \mathrm{Ti}$ ) for $14 \mathrm{~h}$ (Extended Data Fig. 1b and 6a). Prior to further analysis, sucrose was removed via a PD G25 desalting column followed by concentration with a spin column (regenerated cellulose: 100,000 MWCO).

\section{Biochemical analysis of PSI}

For SDS-PAGE (Extended Data Fig. 1c-e), samples were adjusted to $1 \mu \mathrm{g}$ chl, supplemented with loading buffer and incubated at $65{ }^{\circ} \mathrm{C}$ for $15 \mathrm{~min}$. Proteins were separated by $13 \%(\mathrm{w} / \mathrm{v})$ SDS-PAGE ${ }^{34}$. Gels were stained with Coomassie Brilliant Blue R-250 or blotted onto nitrocellulose membranes (Amersham) and detected by specific primary antibodies: PsaF ${ }^{35}$, PC (Agrisera), PsaA, Lhca5, Lhca2, Lhca9 ${ }^{4}$, Lhca3, PsaD ${ }^{36}$, PsaG and LhcB/A ${ }^{37}$. Secondary antibodies for ECL detection were anti-rabbit (Invitrogen). sucrose and protein digestion was carried out following the FASP protocol, using $2 \mu \mathrm{g}$ of sequencing grade trypsin (Promega) per fraction ${ }^{38}$. Iodoacetamide and dithiothreitol used in the original protocol were replaced by chloroacetamide and tris (2-carboxyethyl) phosphine, respectively. After over-night digestion at $37^{\circ} \mathrm{C}$, samples were acidified by adding trifluoroacetic acid (TFA) to a final volume of $0.1 \%$. Five percent of the peptide solution were desalted using self-made Stage tips according to established protocols ${ }^{39}$. Desalted peptides were dried by vacuum centrifugation and stored at $-20^{\circ} \mathrm{C}$ until further use. The LC-MS/MS system consisted of an Ultimate 3000 RSLC nanoLC System (Thermo Fisher Scientific) coupled via a Nanospray Flex ion source (Thermo Fisher Scientific) to a Q Exactive Plus mass spectrometer (Thermo Fisher Scientific). Samples were reconstituted in $5 \mu 1$ of $2 \%$ (v/v) acetonitrile/0.05\% (v/v) TFA in ultrapure water (eluent A1), loaded on a trap column (C18 PepMap 100, $300 \mu \mathrm{M}$ x $5 \mathrm{~mm}, 5$ $\mu \mathrm{m}$ particle size, $100 \AA$ pore size; Thermo Fisher Scientific) and desalted for $3 \mathrm{~min}$ at a flow rate of $15 \mu \mathrm{L} \mathrm{min}{ }^{-1}$ using eluent A1. Subsequently, the trap column was switched in-line with an Acclaim PepMap100 reversed phase column $(75 \mu \mathrm{m} \times 50 \mathrm{~cm}, 2 \mu \mathrm{m}$ particle sizes, $100 \AA$ pore size; Thermo Fisher Scientific) for peptide separation. The mobile phases were composed of 0.1 $\%(\mathrm{v} / \mathrm{v})$ formic acid in ultrapure water (eluent A2) and $80 \%(\mathrm{v} / \mathrm{v})$ acetonitrile $/ 0.08 \%(\mathrm{v} / \mathrm{v})$ formic acid in ultrapure water (B). Flow rate was $250 \mathrm{~nL} / \mathrm{min}$. The following gradient was applied: 5-35 \% B over $105 \mathrm{~min}, 35-99 \%$ B over $5 \mathrm{~min}, 99 \%$ B for $20 \mathrm{~min}$. MS full scans (scan range $\mathrm{m} / \mathrm{z}$ : $350-1400$, resolution 70,000 at $\mathrm{m} / \mathrm{z} 200$, AGC target value $3 \mathrm{e} 6$, maximum injection time $50 \mathrm{~ms}$ ) were acquired in data-dependent mode, dynamically selecting the 12 most abundant precursor ions for fragmentation by higher-energy C-trap dissociation (HCD, $27 \%$ normalized collision energy, resolution 17,500 at $\mathrm{m} / \mathrm{z} 200$, precursor isolation window $1.5 \mathrm{~m} / \mathrm{z}$ ). Dynamic exclusion was set to 'auto' (chromatographic peak width: $15 \mathrm{~s}$ ). AGC target value and intensity threshold for MS/MS were 5e4 and 1e4, respectively, at $80 \mathrm{~ms}$ maximum ion fill time. Singly 
charged ions, ions with charge state 5 or above and ions with unassigned charge states were rejected. Internal lock mass calibration was enabled on $\mathrm{m} / \mathrm{z} 445.12003$. LC-MS/MS data was processed in MaxQuant 1.6.14 for protein identification and label-free quantification ${ }^{40}$. Default settings were used, except for calculation of iBAQ values and "second peptide search" which were enabled and disabled, respectively. Spectra were searched against a concatenated database containing protein sequences based on the Chlamydomonas v5.6 gene models (Joint Genome Institute, www.phytozome.org), as well as sequences of chloroplast- and mitochondrial-encoded proteins (GenBank BK000554.2 and NC_001638.1). Carbamidomethylation of cysteines was set as a fixed modification. Oxidation of methionine and acetylation of protein N-termini were considered as variable modifications. A false discovery rate (FDR) of 1\% was applied to peptide and protein identifications. LFQ data was imported into Perseus (version 1.6.15.0) ${ }^{41}$ for $\log 2$ transformation, and contaminants, proteins only identified by site and reverse hits were removed. iBAQ values were normalized to the PSI core subunit PsaB. Room temperature absorption spectra $(300-750 \mathrm{~nm})$ of PSI monomer and dimer fractions (Extended Data Fig. 1g) were measured with a UV-/Vis spectrophotometer (V-650, Jasco) at 10 $\mu \mathrm{g} \mathrm{chl} \mathrm{mL} \mathrm{m}^{-1}$ and normalized to the red region. Fluorescence emission spectra $(650-780 \mathrm{~nm})$ of PSI fractions (Extended Data Fig. 1h) were recorded at $77 \mathrm{~K}$ at $1 \mu \mathrm{g}$ chl $\mathrm{mL}^{-1}$ with a spectrofluorometer (P-6500, Jasco) upon excitation at $436 \mathrm{~nm}$. Spectra were normalized to the maximum of the emission peaks and smoothed according to ${ }^{42}$. For 2D-PAGE (Extended Data

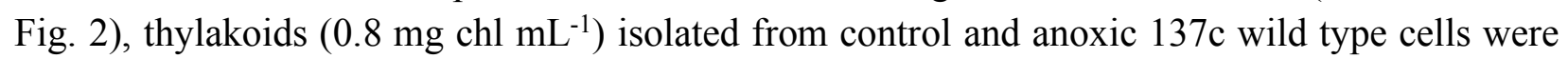
solubilized with $0.9 \% \beta$-DDM for 20 min. 2 D-PAGE ${ }^{43}$, silver staining, tryptic in-gel digestion ${ }^{37}$ and LC-MS/MS was operated as described ${ }^{36}$, where a Ultimate 3000 nano-LC system was coupled via a nanospray source to an LCQ Deca XP mass spectrometer (Thermo Finnigan). LC-MS/MS data were processed with Proteome Discoverer (Thermo Fisher Scientific, version 2.4). Raw files were searched using the SequestHT and MS Amanda algorithms against a concatenated database containing sequences of nuclear- (Chlamydomonas v5.6 gene models, www.phytozome.org), chloroplast- (GenBank BK000554.2) and mitochondrial-encoded (NC_001638.1) proteins Search settings were: precursor and fragment mass tolerances $250 \mathrm{ppm}$ and $0.25 \mathrm{Da}$, respectively; minimum peptide length: 6; maximum of missed cleavages: 2; variable modifications: oxidation of methionine, $\mathrm{N}$-acetylation of protein $\mathrm{N}$-termini. Identifications were

\section{Cryo-EM data collection and processing}

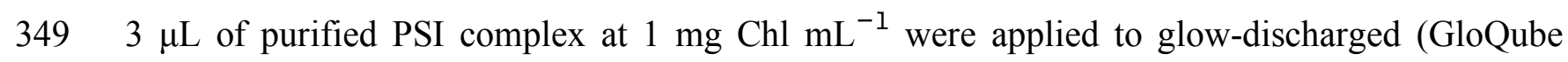
350 Quorum, $40 \mathrm{sec}, 20 \mathrm{~mA}$ ) holey carbon grids (Quantifoil $300 \mathrm{Au}$ R1.2/R1.3, Electron Microscopy Sciences) and vitrified using a Vitrobot MKIV (2 sec blotting time, $4^{\circ} \mathrm{C}, 100 \%$ humidity). The data collection was carried out using a 300KV Titan Krios G2 Microscope (Thermo Fisher Scientific) equipped with a Gatan Bioquantum energy filter and a K3 Summit direct electron detector (Ametek). Movies were recorded using counting mode at a magnification of $\times 105,000$ corresponding to a calibrated pixel size of $0.84 \AA$. The dose rate was $15.27 \mathrm{e} / \mathrm{px} / \mathrm{sec}$ and the exposure time was $3 \mathrm{sec}$ divided into 45 frames leading to a total dose of 45.8 e $\AA^{-2}$. EPU was 
used to collect 17,439 movies with a defocus range from -0.7 to $-2.5 \mu \mathrm{m}$. Data statistics is shown in Supplementary Table 2.

The Extended Data Fig. 3 shows the processing scheme applied. The pre-processing steps were performed using cryoSPARC 3.1.0 ${ }^{44}$. Movie stacks were motion corrected and dose weighted using MotionCor $2{ }^{45}$. Contrast Transfer Function (CTF) of the motion corrected micrographs was estimated using CTFFIND4 ${ }^{46}$. Blob picker and then template picker were used to pick 440,494 particles and 2D classification in cryoSPARC was performed. Dimeric particles were separated from monomeric by inspection of the 2D-class averages and for each sub-population an $A b$ initio model was generated using cryoSPARC applying $\mathrm{C} 2$ and $\mathrm{C} 1$ symmetry, respectively. For each model homogenous refinement was performed leading to a nominal resolution of $3.7 \AA$ for the dimer and $3.0 \AA$ for the monomer. Particles (dimer: 69,144 monomer: 123,746) were converted into a Star file format ${ }^{47}$ and were imported into RELION 3.1.beta ${ }^{48,49}$. Particles were reextracted (un-binned) and processed in RELION using a box size of 700 pixel and 500 pixel for the dimer and monomer, respectively. 3D Refinement followed by 3D classification was performed imposing $\mathrm{C} 2$ symmetry for the dimer and $\mathrm{C} 1$ for the monomer. A subset of high quality particles was selected for the dimer and monomer and subjected to $3 \mathrm{D}$ refinement which resulted in a resolution of $3.3 \AA$ for the dimer and $2.9 \AA$ for the monomer. CTF refinement ${ }^{50,51}$ followed by $3 \mathrm{D}$ refinement and Bayesian polishing followed by another round of CTF refinement was performed for the dimer as well as for the monomer. A final 3D refinement resulted in an overall resolution of $2.97 \AA$ for the dimer and $2.31 \AA$ for the monomer. The resolution of the dimer could be further improved to $2.74 \AA$ by using signal subtraction of one monomer followed by symmetry expansion and $3 \mathrm{D}$ refinement applying $\mathrm{C} 1$ symmetry.

In order to increase the number of particles for classification on the Pc region, Dataset 2 was collected from the same dimer band, but with a pixel size of $0.51 \AA$. The dataset was processed with cryoSPARC 3.1.0 ${ }^{44}$. After template picking 864,399 particles were extracted. With a small subset of the extracted particles an $A b$ initio reconstruction was generated followed by Heterogeneous Refinement using 5 classes, one of which contained $A b$ initio reconstruction as reference. The class containing the PSI monomer was then subjected to Homogeneous Refinement in cryoSPARC 3.1.0 resulting in a reconstruction at $3.88 \AA$ resolution. The particles were then exported to RELION ${ }^{47}$ and $3 \mathrm{D}$ classification was performed. The class that contained 88,219 good particles was used for further refinement which improved the overall resolution to 3.5 A. Applying CTF-refinement and Bayesian Polishing resulted in further improvement, and the final nominal resolution is $2.68 \AA$. The data was then merged with the monomer (Dataset 1) and signal subtraction followed by focused classification using a mask around the Pc region was performed. A class with 66,080 particles showed the best density for Pc which was used for

393 For analysis of the motion between the two monomers of the dimer we performed a Multibody 394 Refinement in RELION ${ }^{52}$ followed by a principal component analysis using the program relion_flex_analyse. Two bodies were chosen, one for each monomer, resulting in twelve Eigenvectors describing the motion. Ten maps for each of the three Eigenvectors which describe 
about $78 \%$ of the motion in the data were printed out and the maps with the extreme positions (map1 and 10) were used to fit the models that are shown in Extended Data Figure 8. A Python script was used to estimate the distances between the chlorophylls at the dimer interface for each particle in the data and to plot the results as histograms as depicted in Extended Data Figure 8e,f.

\section{Model building and refinement}

403 Initially, the available model of the PSI structure (PDB ID: 6JO5) of $C$. reinhardtii was rigid 404 body fitted into the $2.74 \AA$ map of the symmetry expanded dimer using Chimera v $1.14^{53}$. Model building and real-space refinement was then carried out using Coot v9.1.4 ${ }^{26,27}$ to complete one monomer. Two copies of the completed monomeric model were then rigid body fitted into the $\mathrm{C} 2$ generated $2.97 \AA$ dimer map using Chimera. The model of the monomer was then fitted separately in the highest resolution $2.3 \AA$ map of the monomer. All protein residues as well as pigments were fitted using Coot with locally optimized map weights. The cis-trans isomerism of each pigment was judged based on density and modeled accordingly. Newly identified chlorophylls and carotenoids were modeled, when the experimental evidence (density map) supported and the chemical environment matched the surrounding of the pigments. For carotenoids, the density that clearly showed the characteristics of an elongated tetraterpenoid with densities for the four methyl groups, two sticking out on each side of the chain, was identified as a new carotenoid binding site. To further analyze the identity of the corresponding pigment possible candidates were fitted and compared. A carotenoid that fitted best in terms of density and chemical environment was then selected. In case of luteins, particularly the oxygen of the cyclohexane-ring was the main criterion for pigment identity, because it is involved in hydrogen bonding. For the $\mathrm{Chl} b$ identification the densities for the aldehyde group needed to be present as well as the hydrogen bonding occurring with a water molecule that are usually stabilized by other chlorophylls, lipids, and protein side chains. Water molecules involved in pigment interactions were placed manually. All other water molecules were picked by Coot with the auto picking function followed by manual inspection and correction. All high resolution features were modeled using Coot until the model was completed. For all modeling steps restraint files for pigments and ligands were used which were generated using the Grade server (http://grade.globalphasing.org). Restraint files were adopted manually if it was required. fitted using Chimera. Rotamers were corrected for the residues that were allowed due to the better local densities. Self-restraints in Coot were activated followed by flexible fitting into the density. All models were refined using Real-Space-Refine from the PHENIX suite ${ }^{55}$ using the Grade server restraint files for the ligands and a distance .edit file which was generated by Readyset in PHENIX. Further, hydrogen atoms were added for refinement to the model using Readyset. The refinement protocol was optimized using different weight parameters. The refinement statistics are shown in Supplementary Table 2. Multiple rounds of validation and model building were carried out using MolProbity ${ }^{56}$ and Coot. For further validation the PDB Validation server 
The structure was analyzed using Coot and Chimera. Figures were prepared using ChimeraX ${ }^{57}$.

\section{Data availability}

The atomic coordinates have been deposited in the Protein Data Bank (PDB) and are available under the accession codes: PDB ID: XXX for the dimer, PDB ID: XXXX for the symmetry expanded dimer, PDB ID: XXXX for the high resolution monomer, and PDB ID: XXXX for the PSI-Pc model. The local resolution filtered maps, half maps, masks and FSC-curves have been deposited in the Electron Microscopy Data Bank with the accession codes EMD-XXXXX for the dimer, EMD-XXXXX for the symmetry expanded dimer, EMD-XXXXX for the high resolution monomer, and EMD-XXXXX for the Plastocyanin map. Mass spectrometry datasets: Project Name: Intensity-based absolute quantification (iBAQ) of components of photosystem I monomers and dimers. Project accession: PXD026990. Project DOI: 10.6019/PXD026990. Project Name: Identification of photosystem I components from Chlamydomonas reinhardtii grown under oxic and anoxic conditions. Project accession: PXD027067.

\section{References}

1 Qin, X. et al. Structure of a green algal photosystem I in complex with a large number of light-harvesting complex I subunits. Nat Plants 5, 263-272, doi:10.1038/s41477-0190379-y (2019).

2 Suga, M. et al. Structure of the green algal photosystem I supercomplex with a decameric light-harvesting complex I. Nat Plants 5, 626-636, doi:10.1038/s41477-019-0438-4 (2019).

$3 \mathrm{Su}, \mathrm{X}$. et al. Antenna arrangement and energy transfer pathways of a green algal photosystem-I-LHCI supercomplex. Nat Plants 5, 273-281, doi:10.1038/s41477-0190380-5 (2019).

4 Ozawa, S. I. et al. Configuration of Ten Light-Harvesting Chlorophyll a/b Complex I Subunits in Chlamydomonas reinhardtii Photosystem I. Plant Physiol 178, 583-595, doi:10.1104/pp.18.00749 (2018).

$5 \quad$ Huang, Z. et al. Structure of photosystem I-LHCI-LHCII from the green alga Chlamydomonas reinhardtii in State 2. Nat Commun 12, 1100, doi:10.1038/s41467-02121362-6 (2021).

6 Pan, X. et al. Structural basis of LhcbM5-mediated state transitions in green algae. Nat Plants, doi:10.1038/s41477-021-00960-8 (2021).

7 Chen, M. et al. Distinct structural modulation of photosystem I and lipid environment stabilizes its tetrameric assembly. Nat Plants 6, 314-320, doi:10.1038/s41477-020-0610-x (2020).

8 Kato, K. et al. Structure of a cyanobacterial photosystem I tetramer revealed by cryoelectron microscopy. Nat Commun 10, 4929, doi:10.1038/s41467-019-12942-8 (2019).

9 Zheng, L. et al. Structural and functional insights into the tetrameric photosystem I from heterocyst-forming cyanobacteria. Nat Plants 5, 1087-1097, doi:10.1038/s41477-0190525-6 (2019). 
$47910 \quad$ Jordan, P. et al. Three-dimensional structure of cyanobacterial photosystem I at $2.5 \mathrm{~A}$ resolution. Nature 411, 909-917, doi:10.1038/35082000 (2001).

$481 \quad 11$ Ben-Shem, A., Frolow, F. \& Nelson, N. Crystal structure of plant photosystem I. Nature 426, 630-635, doi:10.1038/nature02200 (2003).

12 Amunts, A. \& Nelson, N. Plant photosystem I design in the light of evolution. Structure 17, 637-650, doi:10.1016/j.str.2009.03.006 (2009).

13 Perez-Boerema, A. et al. Structure of a minimal photosystem I from the green alga Dunaliella salina. Nat Plants 6, 321-327, doi:10.1038/s41477-020-0611-9 (2020).

14 Wood, W. H. J. et al. Dynamic thylakoid stacking regulates the balance between linear and cyclic photosynthetic electron transfer. Nat Plants 4, 116-127, doi:10.1038/s41477017-0092-7 (2018).

15 Yadav, K. N. et al. Supercomplexes of plant photosystem I with cytochrome b6f, lightharvesting complex II and NDH. Biochim Biophys Acta Bioenerg 1858, 12-20, doi:10.1016/j.bbabio.2016.10.006 (2017).

16 Mazor, Y., Borovikova, A., Caspy, I. \& Nelson, N. Structure of the plant photosystem I supercomplex at 2.6 A resolution. Nat Plants 3, 17014, doi:10.1038/nplants.2017.14 (2017).

17 Wang, J. et al. Structure of plant photosystem I-light harvesting complex I supercomplex at 2.4 A resolution. J Integr Plant Biol, doi:10.1111/jipb.13095 (2021).

18 Muhleip, A., McComas, S. E. \& Amunts, A. Structure of a mitochondrial ATP synthase with bound native cardiolipin. Elife 8, doi:10.7554/eLife.51179 (2019).

19 Flygaard, R. K., Muhleip, A., Tobiasson, V. \& Amunts, A. Type III ATP synthase is a symmetry-deviated dimer that induces membrane curvature through tetramerization. Nat Commun 11, 5342, doi:10.1038/s41467-020-18993-6 (2020). mitochondria. Nat Commun 12, 120, doi:10.1038/s41467-020-20381-z (2021).

21 Bennett, C. F. et al. Peroxisomal-derived ether phospholipids link nucleotides to respirasome assembly. Nat Chem Biol, doi:10.1038/s41589-021-00772-z (2021).

22 Pan, X. et al. Structure of the maize photosystem I supercomplex with light-harvesting complexes I and II. Science 360, 1109-1113, doi:10.1126/science.aat1156 (2018). Amunts, A., Toporik, H., Borovikova, A. \& Nelson, N. Structure determination and improved model of plant photosystem I. J Biol Chem 285, 3478-3486, doi:10.1074/jbc.M109.072645 (2010). characterization of a large photosystem I-light-harvesting complex II supercomplex with an additional Lhca1-a4 dimer in Arabidopsis. Plant J 102, 398-409, doi:10.1111/tpj.14634 (2020).

25 Croce, R. \& van Amerongen, H. Light harvesting in oxygenic photosynthesis: Structural biology meets spectroscopy. Science 369, doi:10.1126/science.aay2058 (2020). Acta Crystallogr D Biol Crystallogr 66, 486-501, doi:10.1107/S0907444910007493 (2010).

28 Caspy, I., Borovikova-Sheinker, A., Klaiman, D., Shkolnisky, Y. \& Nelson, N. The structure of a triple complex of plant photosystem I with ferredoxin and plastocyanin. Nat Plants 6, 1300-1305, doi:10.1038/s41477-020-00779-9 (2020). 
29 Caspy, I. et al. Structure of plant Photosystem I-plastocyanin complex reveals strong hydrophobic interactions. Biochem J, doi:10.1042/BCJ20210267 (2021).

30 Hippler, M., Drepper, F., Haehnel, W. \& Rochaix, J. D. The N-terminal domain of PsaF: precise recognition site for binding and fast electron transfer from cytochrome $\mathrm{c} 6$ and plastocyanin to photosystem I of Chlamydomonas reinhardtii. Proc Natl Acad Sci U S A 95, 7339-7344, doi:10.1073/pnas.95.13.7339 (1998).

31 Ben Fredj, A., Ben Lakhdar, Z. \& Ruiz-Lopez, M. F. The structure of chlorophyll a-water complexes: insights from quantum chemistry calculations. Chem Commun (Camb), 718720, doi:10.1039/b716800d (2008).

$32 \mathrm{Li}, \mathrm{X}$. et al. A genome-wide algal mutant library and functional screen identifies genes required for eukaryotic photosynthesis. Nat Genet 51, 627-635, doi:10.1038/s41588-0190370-6 (2019).

33 Hoober, J. K. The Chlamydomonas Sourcebook. A Comprehensive Guide to Biology and Laboratory Use. Elizabeth H. Harris. Academic Press, San Diego, CA, 1989. xiv, 780 pp., illus. \$145. Science 246, 1503-1504, doi:10.1126/science.246.4936.1503-a (1989).

34 Laemmli, U. K. Cleavage of structural proteins during the assembly of the head of bacteriophage T4. Nature 227, 680-685, doi:10.1038/227680a0 (1970).

35 Hippler, M., Drepper, F., Farah, J. \& Rochaix, J. D. Fast electron transfer from cytochrome c6 and plastocyanin to photosystem I of Chlamydomonas reinhardtii requires PsaF. Biochemistry 36, 6343-6349, doi:10.1021/bi970082c (1997).

36 Naumann, B., Stauber, E. J., Busch, A., Sommer, F. \& Hippler, M. N-terminal processing of Lhca3 Is a key step in remodeling of the photosystem I-light-harvesting complex under iron deficiency in Chlamydomonas reinhardtii. J Biol Chem 280, 20431-20441, doi:10.1074/jbc.M414486200 (2005).

37 Hippler, M., Klein, J., Fink, A., Allinger, T. \& Hoerth, P. Towards functional proteomics of membrane protein complexes: analysis of thylakoid membranes from Chlamydomonas reinhardtii. Plant J 28, 595-606, doi:10.1046/j.1365-313x.2001.01175.x (2001).

38 Wisniewski, J. R., Zougman, A., Nagaraj, N. \& Mann, M. Universal sample preparation method for proteome analysis. Nat Methods 6, 359-362, doi:10.1038/nmeth.1322 (2009).

39 Rappsilber, J., Mann, M. \& Ishihama, Y. Protocol for micro-purification, enrichment, prefractionation and storage of peptides for proteomics using StageTips. Nature protocols $\mathbf{2}$, 1896-1906, doi:10.1038/nprot.2007.261 (2007).

40 Cox, J. \& Mann, M. MaxQuant enables high peptide identification rates, individualized p.p.b.-range mass accuracies and proteome-wide protein quantification. Nat Biotechnol 26, 1367-1372, doi:10.1038/nbt.1511 (2008).

41 Tyanova, S. et al. The Perseus computational platform for comprehensive analysis of (prote)omics data. Nat Methods 13, 731-740, doi:10.1038/nmeth.3901 (2016).

42 Savitzky, A. \& Golay, M. J. E. Smoothing and Differentiation of Data by Simplified Least Squares Procedures. Analytical Chemistry 36, 1627-1639, doi:10.1021/ac60214a047 (1964).

43 Busch, A., Nield, J. \& Hippler, M. The composition and structure of photosystem Iassociated antenna from Cyanidioschyzon merolae. Plant J 62, 886-897, doi:10.1111/j.1365-313X.2010.04202.x (2010).

44 Punjani, A., Rubinstein, J. L., Fleet, D. J. \& Brubaker, M. A. cryoSPARC: algorithms for rapid unsupervised cryo-EM structure determination. Nat Methods 14, 290-296, doi:10.1038/nmeth.4169 (2017). 
Zheng, S. Q. et al. MotionCor2: anisotropic correction of beam-induced motion for improved cryo-electron microscopy. Nat Methods 14, 331-332, doi:10.1038/nmeth.4193 (2017).

46 Rohou, A. \& Grigorieff, N. CTFFIND4: Fast and accurate defocus estimation from electron micrographs. J Struct Biol 192, 216-221, doi:10.1016/j.jsb.2015.08.008 (2015). Asarnow, D., Palovcak, E. \& Cheng, Y. UCSF pyem v0.5. Zenodo https://doi.org/10.5281/zenodo.3576630. (2019). Scheres, S. H. W. Amyloid structure determination in RELION-3.1. bioRxiv (2019). Zivanov, J., Nakane, T. \& Scheres, S. H. W. Estimation of high-order aberrations and anisotropic magnification from cryo-EM data sets in RELION-3.1. IUCrJ 7, 253-267, doi:10.1107/S2052252520000081 (2020).

50 Zivanov, J. et al. New tools for automated high-resolution cryo-EM structure determination in RELION-3. Elife 7, doi:10.7554/eLife.42166 (2018). motion correction in cryo-EM single-particle analysis. IUCrJ 6, 5-17, doi:10.1107/S205225251801463X (2019).

52 Nakane, T., Kimanius, D., Lindahl, E. \& Scheres, S. H. Characterisation of molecular motions in cryo-EM single-particle data by multi-body refinement in RELION. Elife 7, doi:10.7554/eLife.36861 (2018).

53 Pettersen, E. F. et al. UCSF Chimera--a visualization system for exploratory research and analysis. J Comput Chem 25, 1605-1612, doi:10.1002/jcc.20084 (2004).

54 Waterhouse, A. et al. SWISS-MODEL: homology modelling of protein structures and complexes. Nucleic Acids Res 46, W296-W303, doi:10.1093/nar/gky427 (2018).

55 Afonine, P. V. et al. Real-space refinement in PHENIX for cryo-EM and crystallography. Acta Crystallogr D Struct Biol 74, 531-544, doi:10.1107/S2059798318006551 (2018).

56 Williams, C. J. et al. MolProbity: More and better reference data for improved all-atom structure validation. Protein Sci 27, 293-315, doi:10.1002/pro.3330 (2018).

57 Goddard, T. D. et al. UCSF ChimeraX: Meeting modern challenges in visualization and analysis. Protein Sci 27, 14-25, doi:10.1002/pro.3235 (2018).

\section{Acknowledgements}

The cryo-EM data were collected at the SciLifeLab facility (funded by the KAW, EPS, and Kempe foundations) and the Karolinska Institutet 3D-EM facility. We thank D. Kimanius for providing a script for conversion of poses from Euler space into an orientation matrix in Cartesian space, S. Hawat for mass spectrometric analyses, V. Adlfar for isolation of Pc.

\section{Author contributions}

614 M.H. and A.A. designed the project. L.M., A.P.B. and A.N. prepared the sample for cryo-EM and 615 performed initial screening. A.N. collected and processed the cryo-EM data and built the model. 
616 L.M. and S.K. performed biochemical analysis. V.T. performed computational analysis. Y.T. 617 provided antibodies. A.N. and A.A. analysed the structure and wrote the manuscript with 618 contributions from L.M. and M.H. All authors contributed to the analysis and the final version of 619 the manuscript.

620

\section{Competing interests}

622 The authors declare no competing interests. 
a

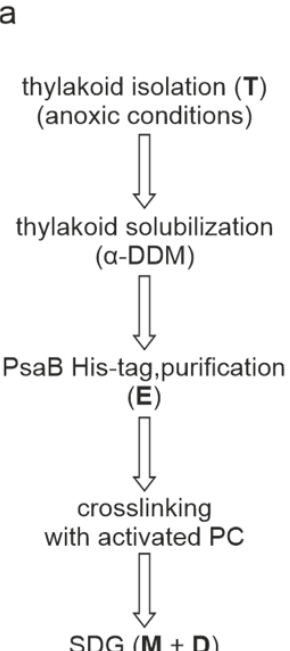

$\operatorname{SDG}(\mathbf{M}+\mathbf{D})$

$f$

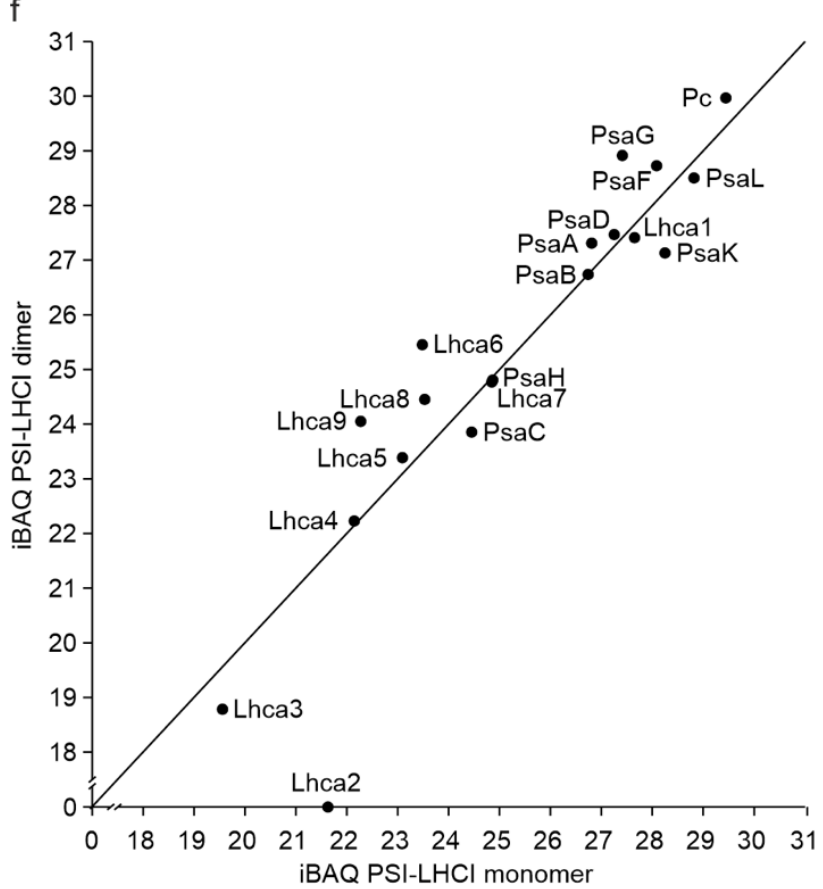

$\mathrm{b}$

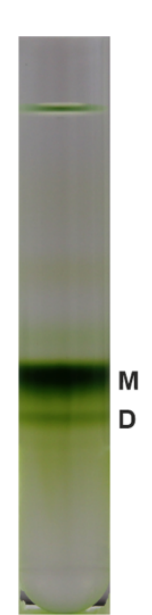

C

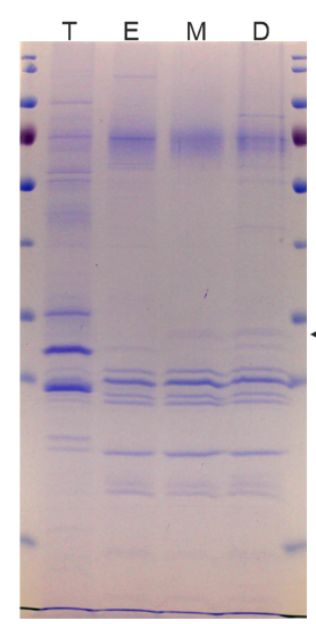

g

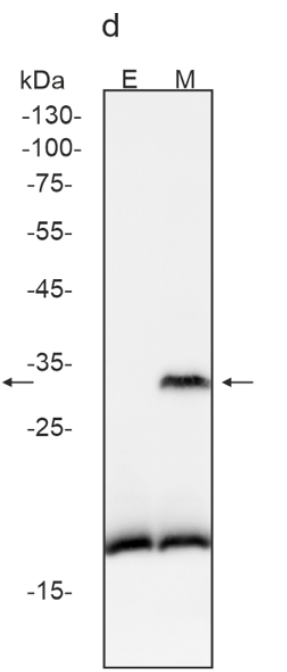

e
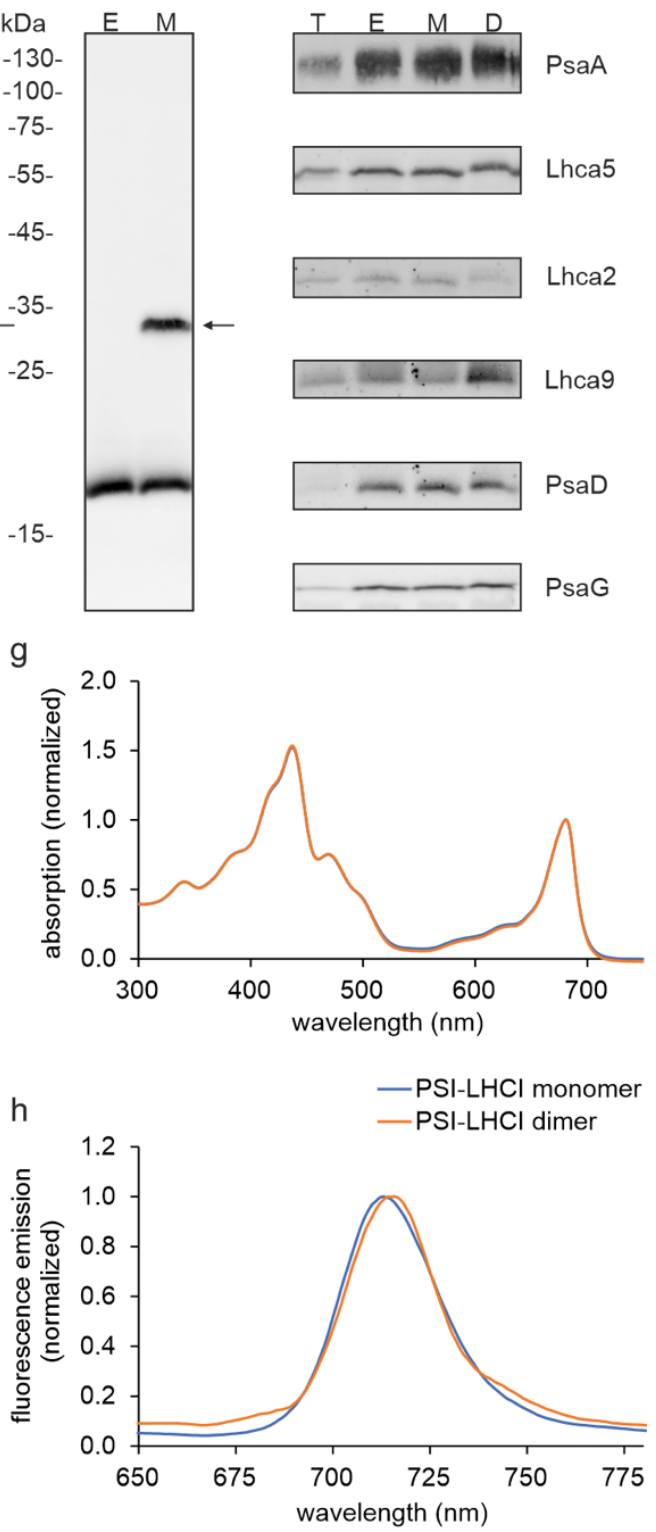

Extended Data Fig. 1: Sample preparation and characterisation. a, The experimental workflow. b, Sucrose density gradient affinity purified PSI $(\sim 60 \mu \mathrm{g}$ chl). Monomer (M) and dimer (D) fractions are indicated. c, SDS-PAGE analysis $(1 \mu \mathrm{g}$ chl). T: thylakoids; E: PSAB-His elution, M: PSI monomer, D: PSI dimer. The arrow indicates a putative PsaF-Pc crosslinked product. d, SDS-PAGE and Western Blot against PsaF confirming the cross-linking with Pc. e, SDS PAGE and Western Blot against PsaA, Lhca5, Lhca2, Lhca9, PsaD and PsaG confirming the presence of small PSI subunits as well as LHCI subunits from both sides of the PSI core. $\mathbf{f}$, Quantitative mass spectrometry analysis of the PSI fractions from (b). iBAQ values are normalized to the PSI core subunit PsaB. g, Room temperature absorption spectra of PSI monomer and dimer fractions. The spectra are normalized to the red region. $\mathbf{h}$, Low temperature $(77 \mathrm{~K})$ fluorescence emission spectra of PSI monomer and dimer fractions upon excitation of chlorophyll $a$ at $436 \mathrm{~nm}$. The spectra are normalized to the maximum of the emission peaks and smoothed according to ${ }^{42}$. 

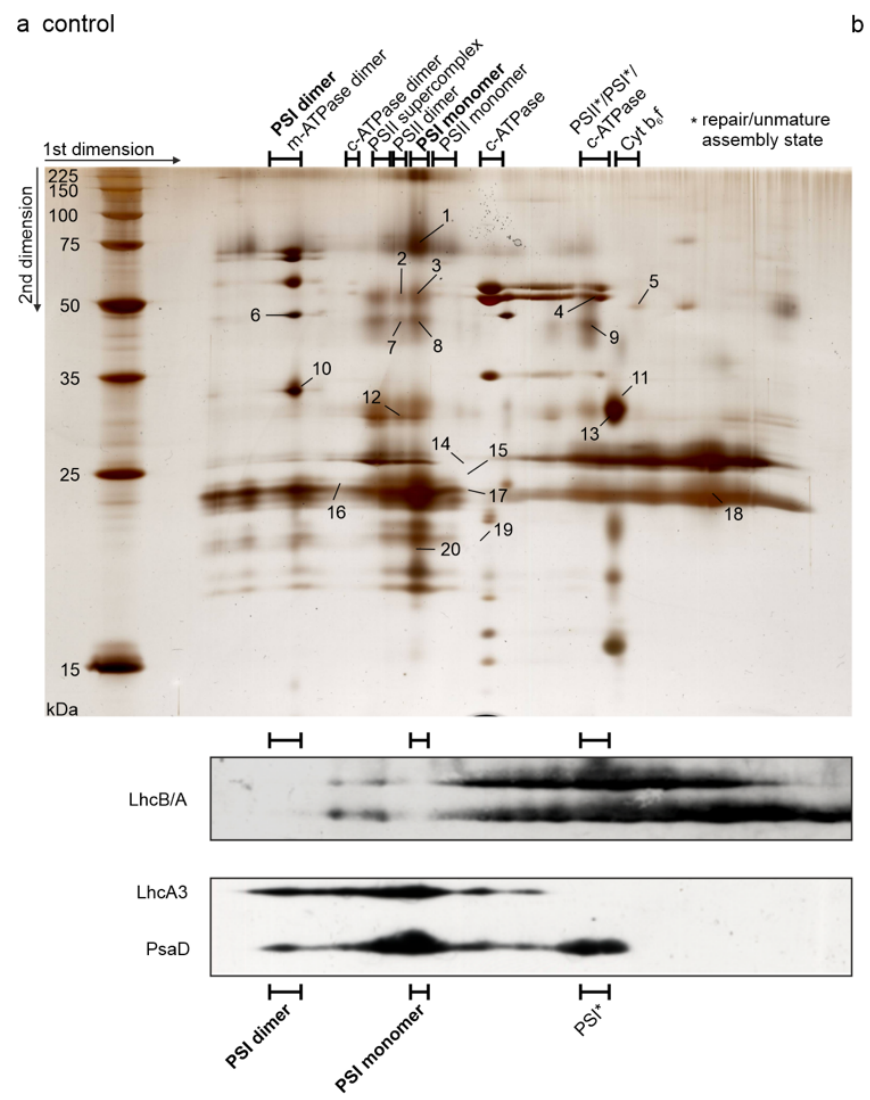

b anoxia
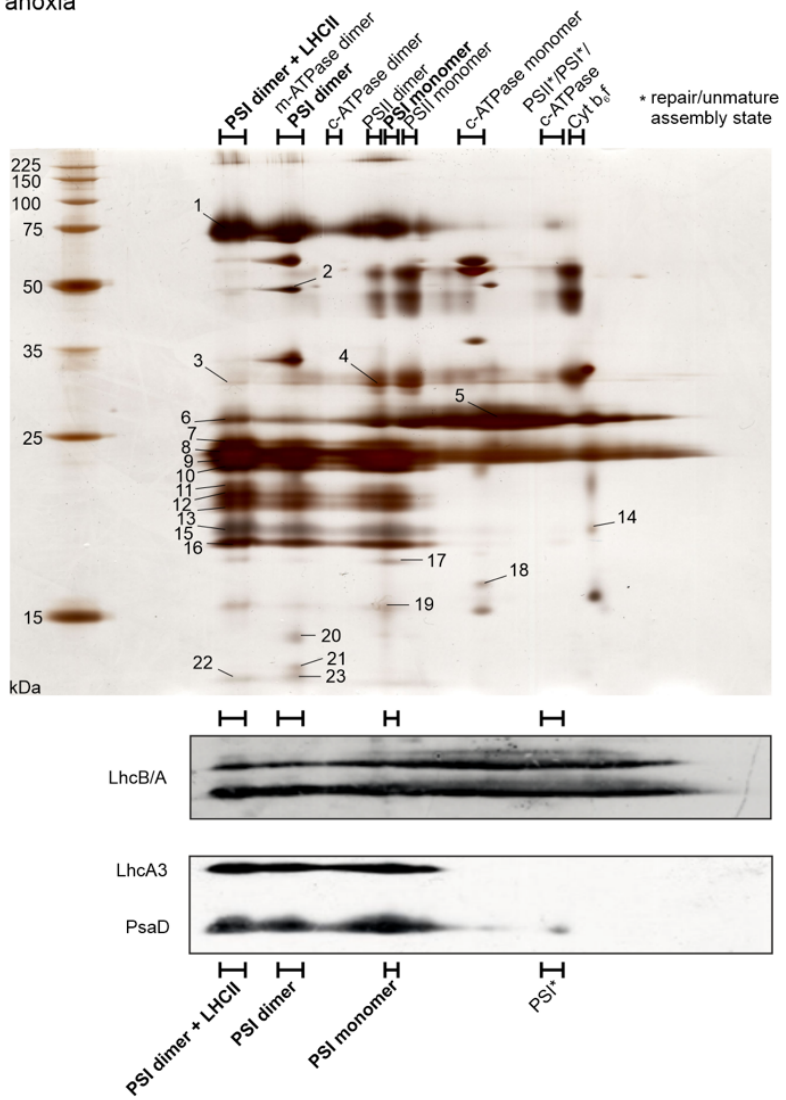

Extended Data Fig. 2: 2D-PAGE of solubilized $C$. reinhardtii wild type thylakoids. a, control conditions. b, low light and anoxic conditions. In the first dimension, multi-protein complexes were separated in their native form by blue native PAGE. In the second dimension, subunits of these protein complexes were separated by SDS-PAGE. Labelled silver-stained spots were subjected to LC-MS/MS analysis. Western Blots of LhcB/A, Lhca3 and PsaD indicate the distribution of LHCII, LHCI and the PSI core. 
bioRxiv preprint doi: https://doi.org/10.1101/2021.08.30.458224; this version posted August 31, 2021. The copyright holder for this preprint (which was not certified by peer review) is the author/funder, who has granted bioRxiv a license to display the preprint in perpetuity. It is made available under aCC-BY 4.0 International license.

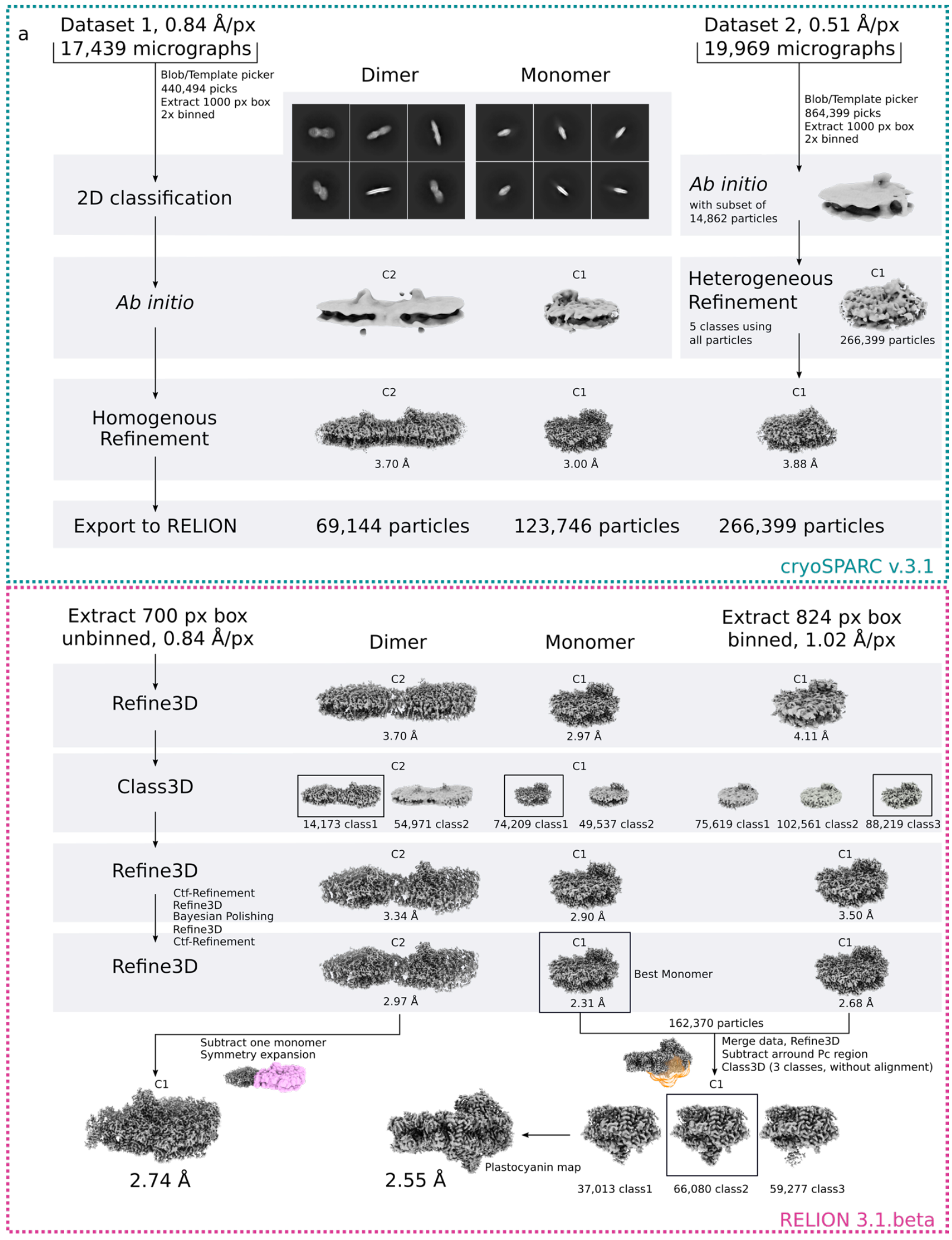


b

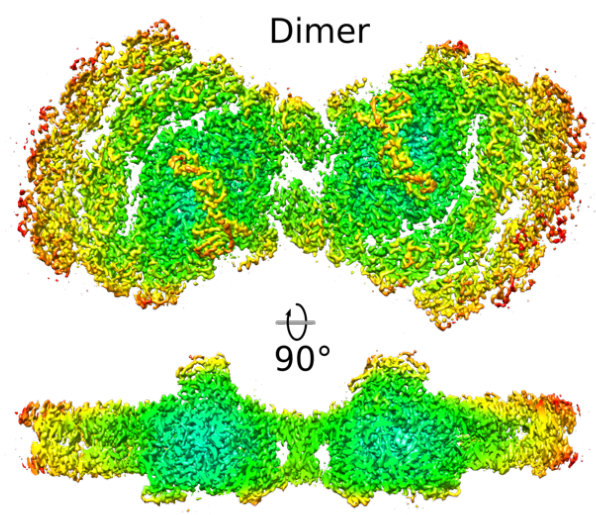

cut-through

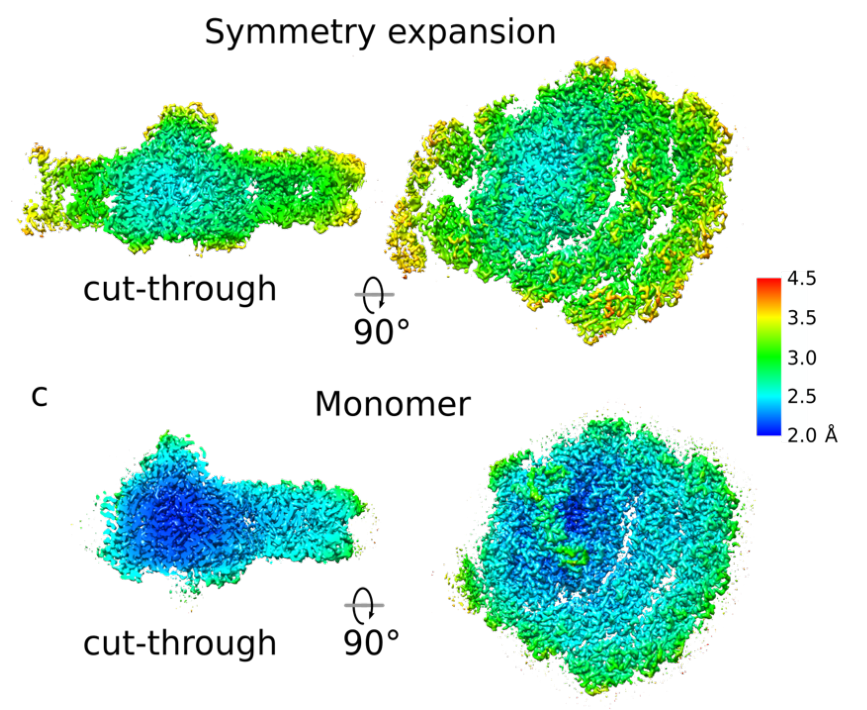

d

Plastocyanin map

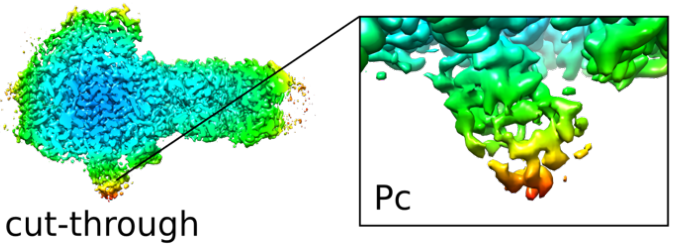

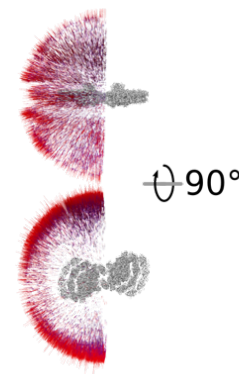
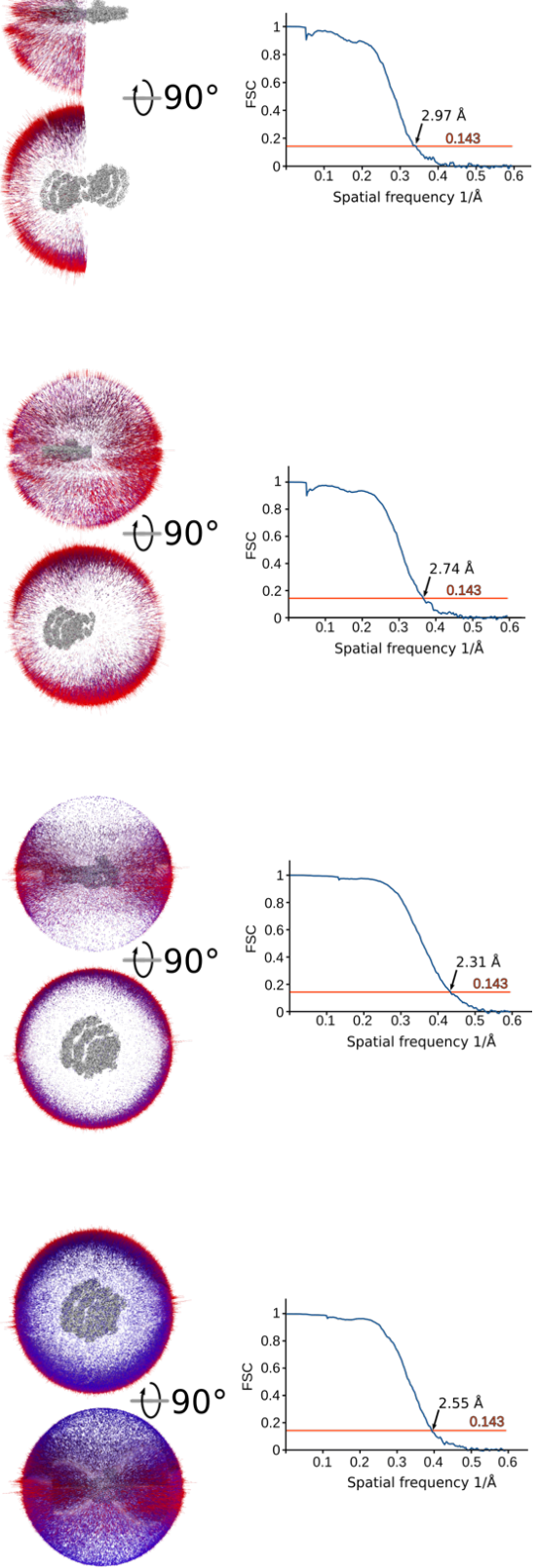

647 Extended Data Fig. 3: Cryo-EM processing workflow and local resolution. a, Flowchart of 648 the data processing for the PSI dimer and monomer. The processing steps performed in 649 cryoSPARC and RELION are indicated. To increase the numbers of Pc bound particles Dataset 2 650 was added for focused classification with signal subtraction. b, The map of the dimer and 651 symmetry expanded dimer colored by local resolution in two orientations (left). Angular 652 distribution of the dimer and symmetry expanded dimer and the FSC-curves are shown. c, The 653 map of the monomer, angular distribution and FSC-curve. d, The map with the best density for 654 plastocyanin, angular distribution and FSC-curve. 


\section{Monomer}

PsaG

Lheag

$132-153$

63-77

(1)
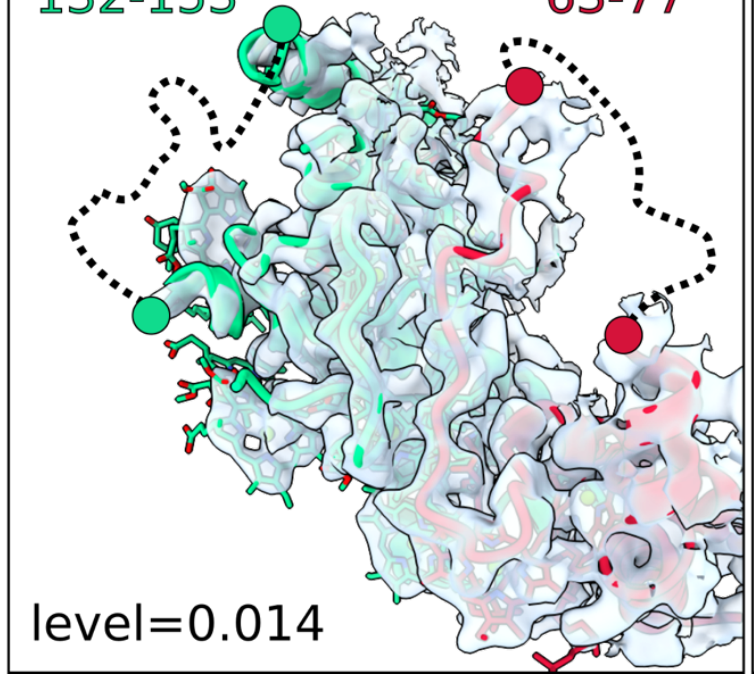

level $=0.02$

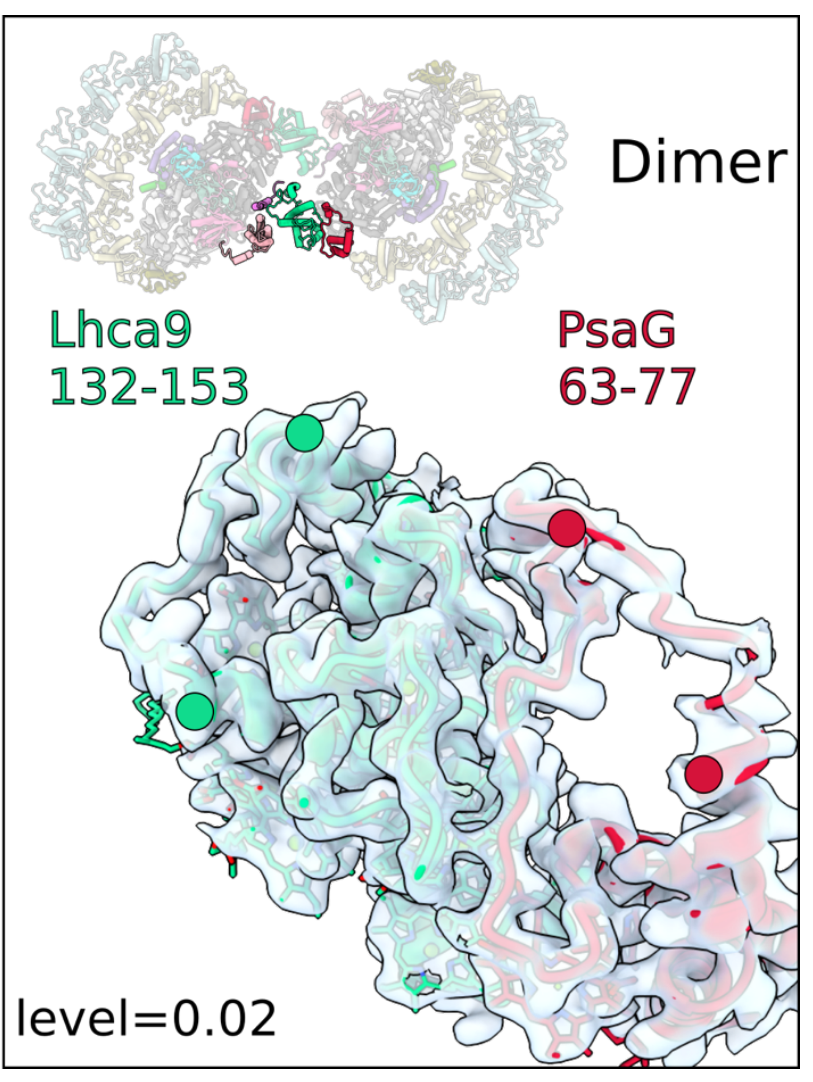

657

Extended Data Fig. 4: Stabilization of loops in Lhca9 and PsaG in PSI dimer. Left, in the monomer, the Lhca9 loop 132-153 and PsaG loop 63-77 are disordered (dashed lines). Right, in the dimer, both loop regions are ordered due to a stabilization by the neighboring monomer. 


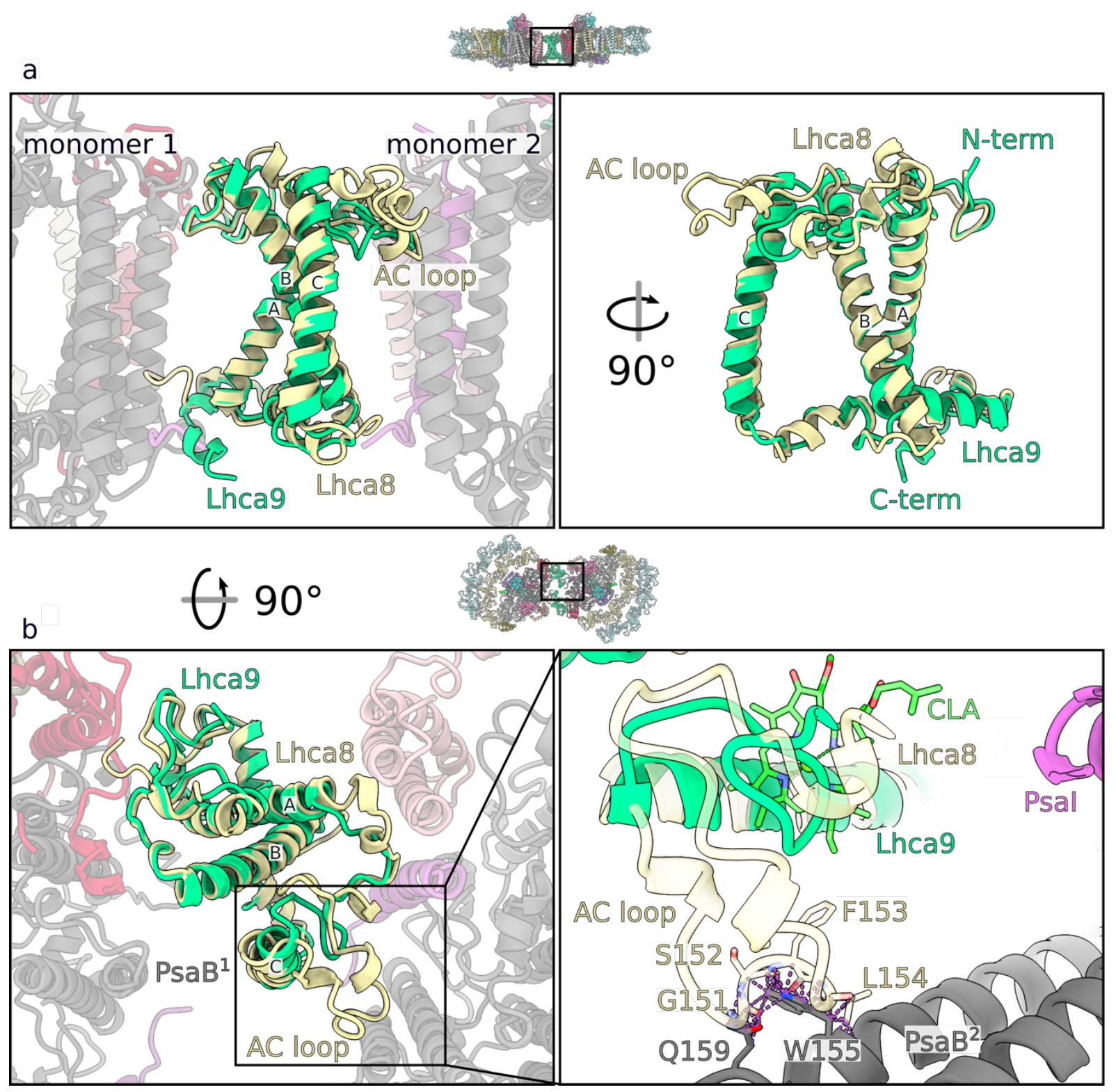

Extended Data Fig. 5: Lhca9 short AC-loop contributes to the dimerization. a, Superposition

663 of Lhca 8 and Lhca9, highlighting the difference in the AC-loop. b, The $90^{\circ}$-rotated view 664 illustrates a clash between the longer AC-loop version and PsaB of monomer 2. Clashes between 665 atoms with a VdW overlap $>0.6 \AA$ are highlighted by the dashed lines. 
a

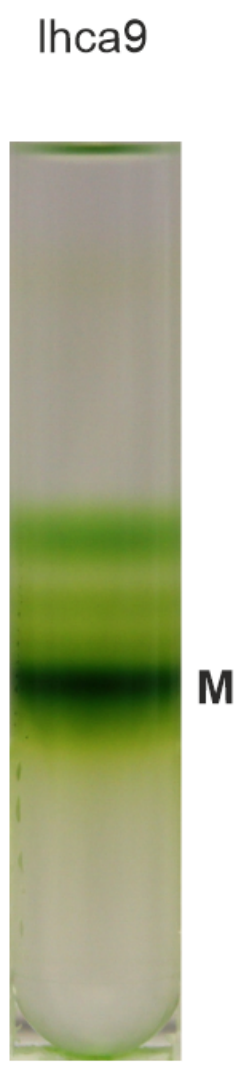

666

667

668

669

670

671 subunit PsaB. b

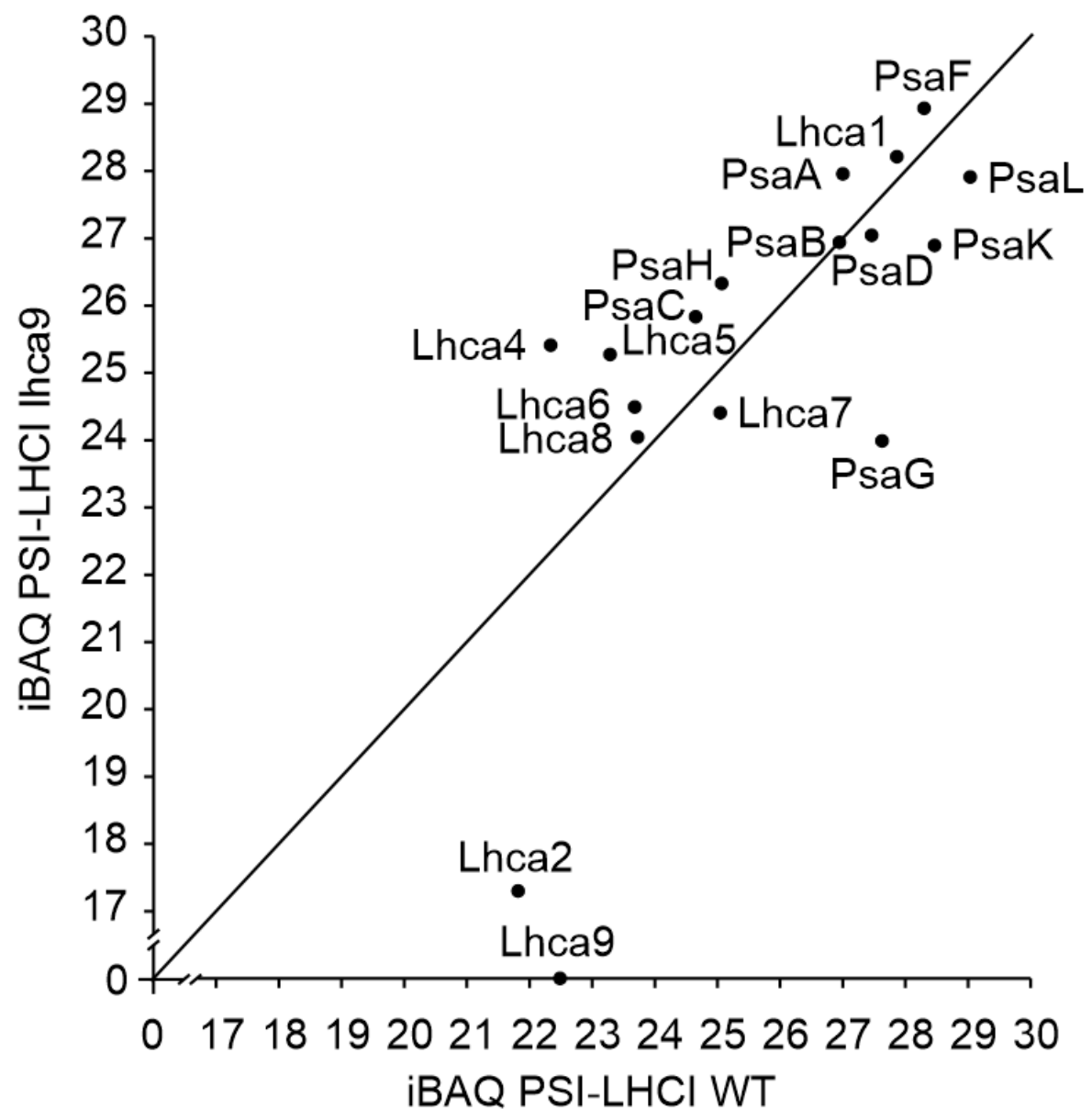

Extended Data Fig. 6: PSI dimer fraction is absent in Alhca9. a, Sucrose density gradient of affinity purified PSI from lhca9 insertional mutant $(\sim 60 \mu \mathrm{g}$ chl $)$. The monomer $(\mathrm{M})$ fraction is indicated. b, Quantitative mass spectrometry analysis of the PSI monomer fraction from (a) compared to wild type (Extended Data Fig. 1). iBAQ values are normalized to the PSI core 
a

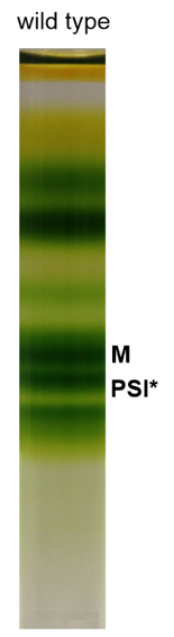

b

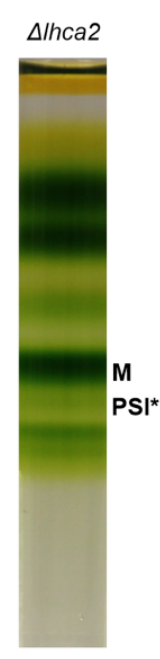

C

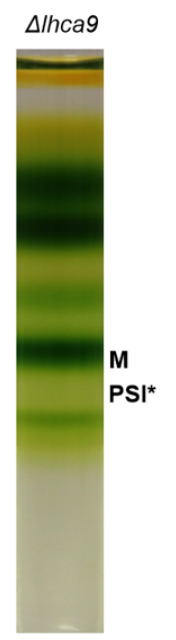

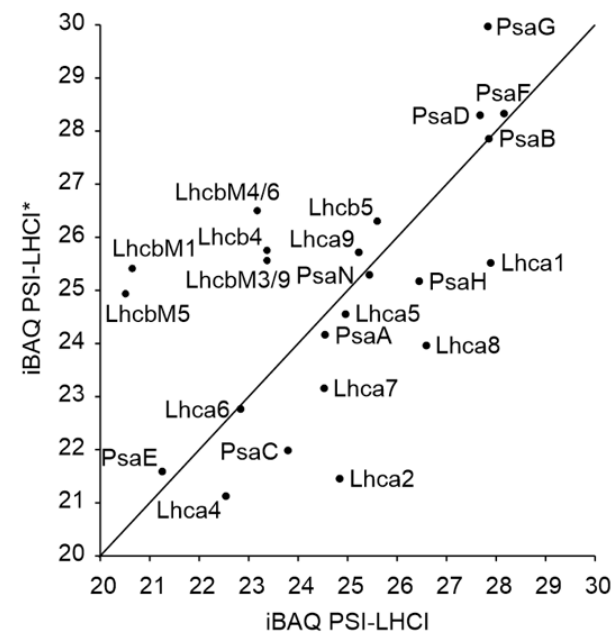
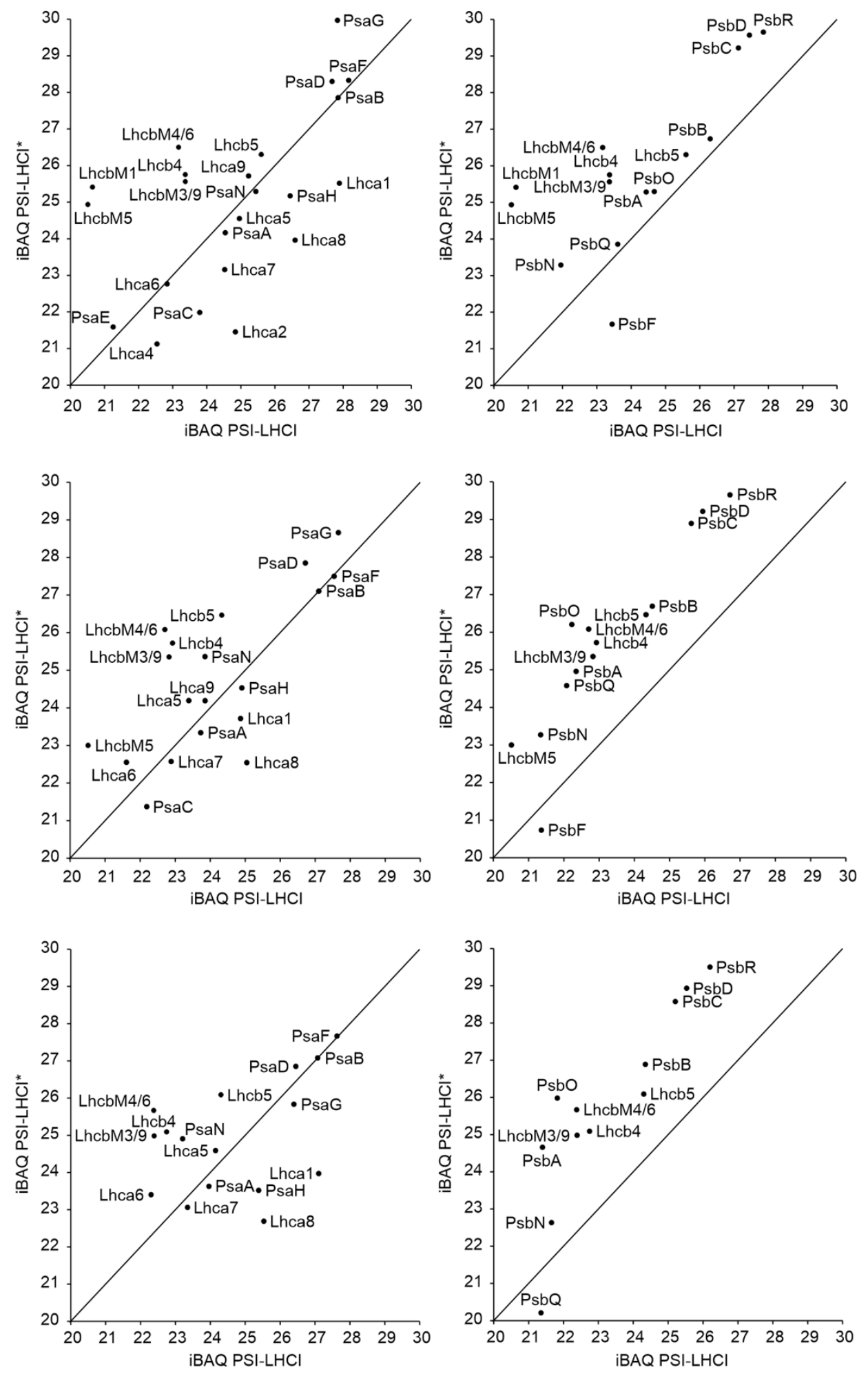

Extended Data Fig. 7: Sucrose density gradient analysis of non-tagged wild type, Alhca2 and Alhca9. Sucrose density gradients of solubilized thylakoids isolated from wild type (a), fractions. iBAQ values are normalized to the PSI core subunit PsaB. 'M' is PSI monomer, 'PSI*' is a lower PSI band. 
a

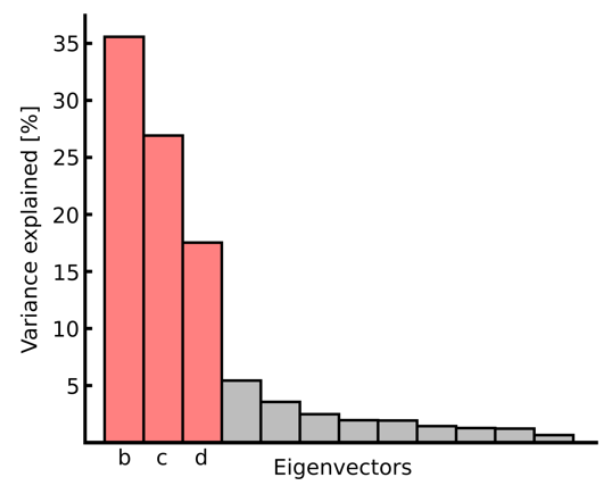

b

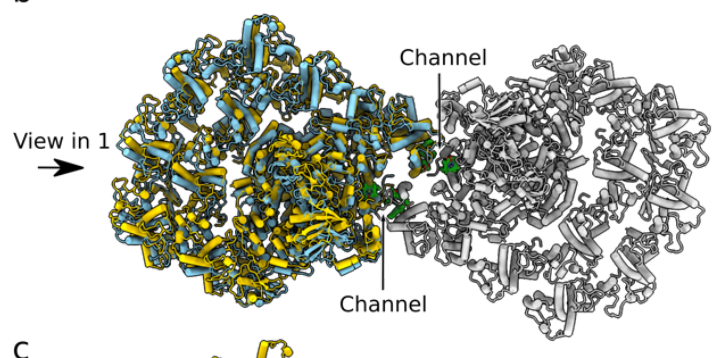

C

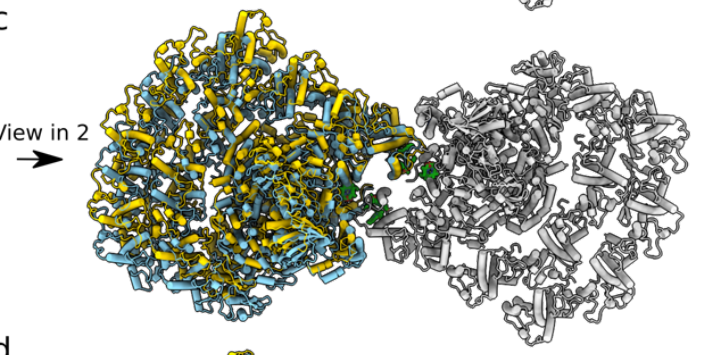

d
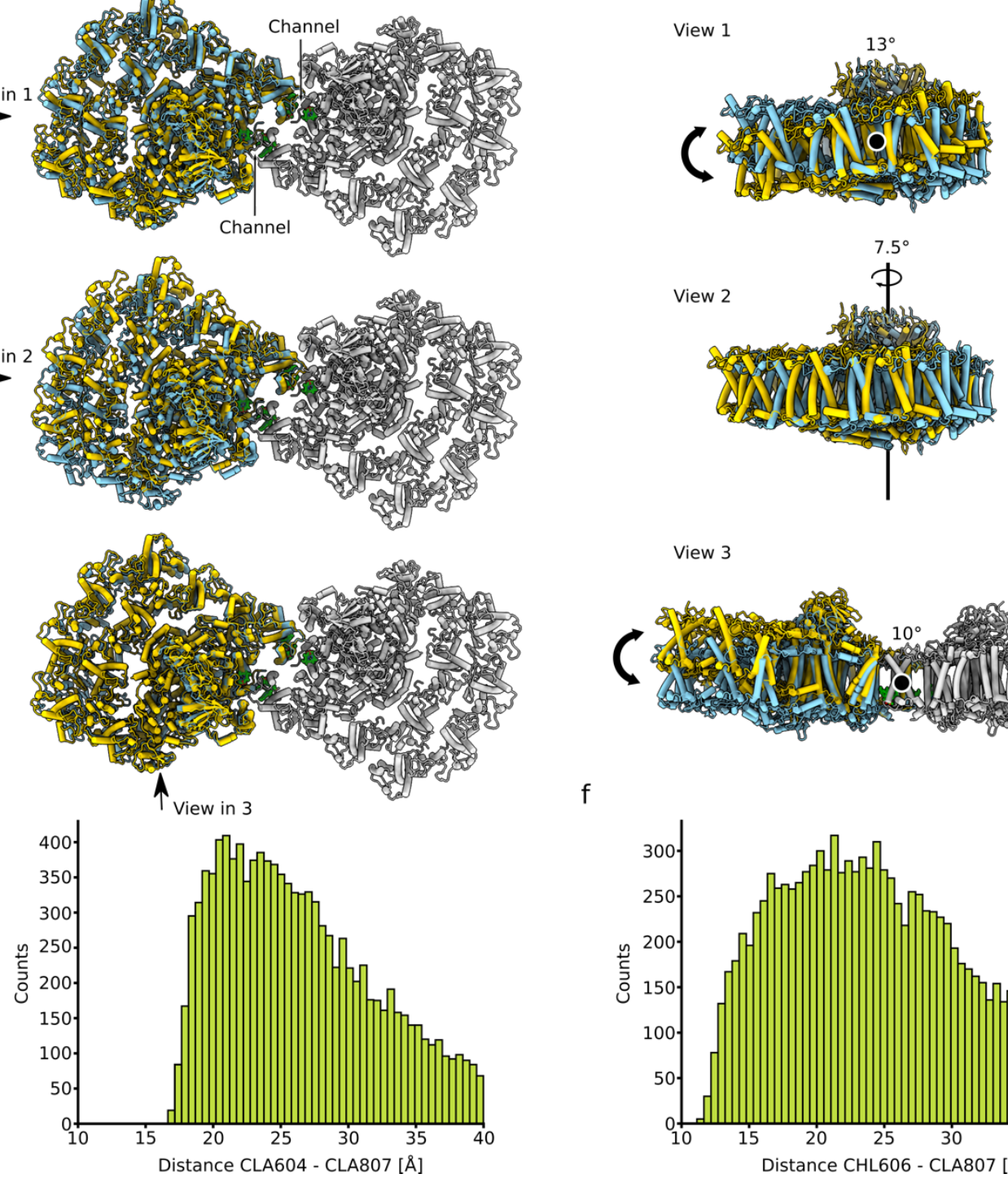

View 3

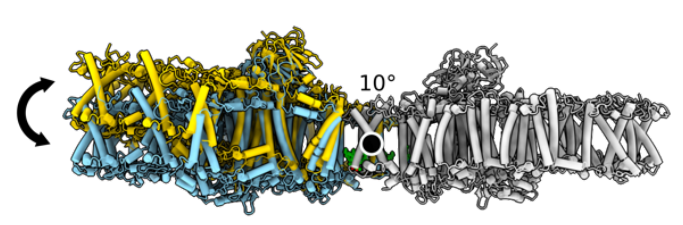

f

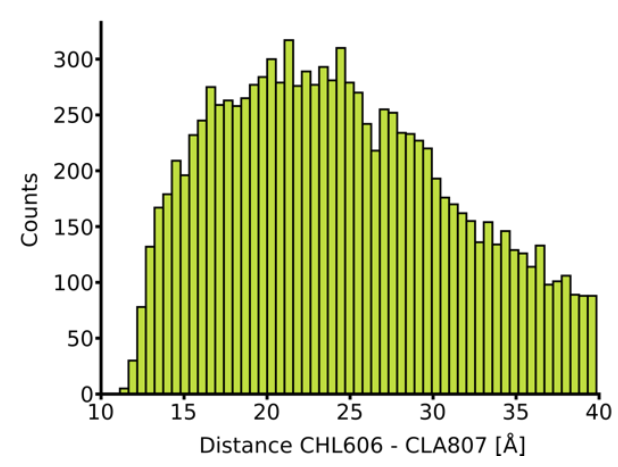

680 Extended Data Fig. 8: Multi-body refinement analysis. a, Eigenvectors that explain the 681 variability of the data. The three major eigenvectors account for $\sim 78 \%$ of the motion in the PSI 682 dimer. b-d, Stromal and side view of the model, showing the maximal motion along the three 683 vectors. e, f, The distance between the chlorophylls is plotted based on the relative motion from 684 the multi-body analysis. The y-axis shows the counts of particles that exhibit a certain distance. 
PDB ID: 7DKZ

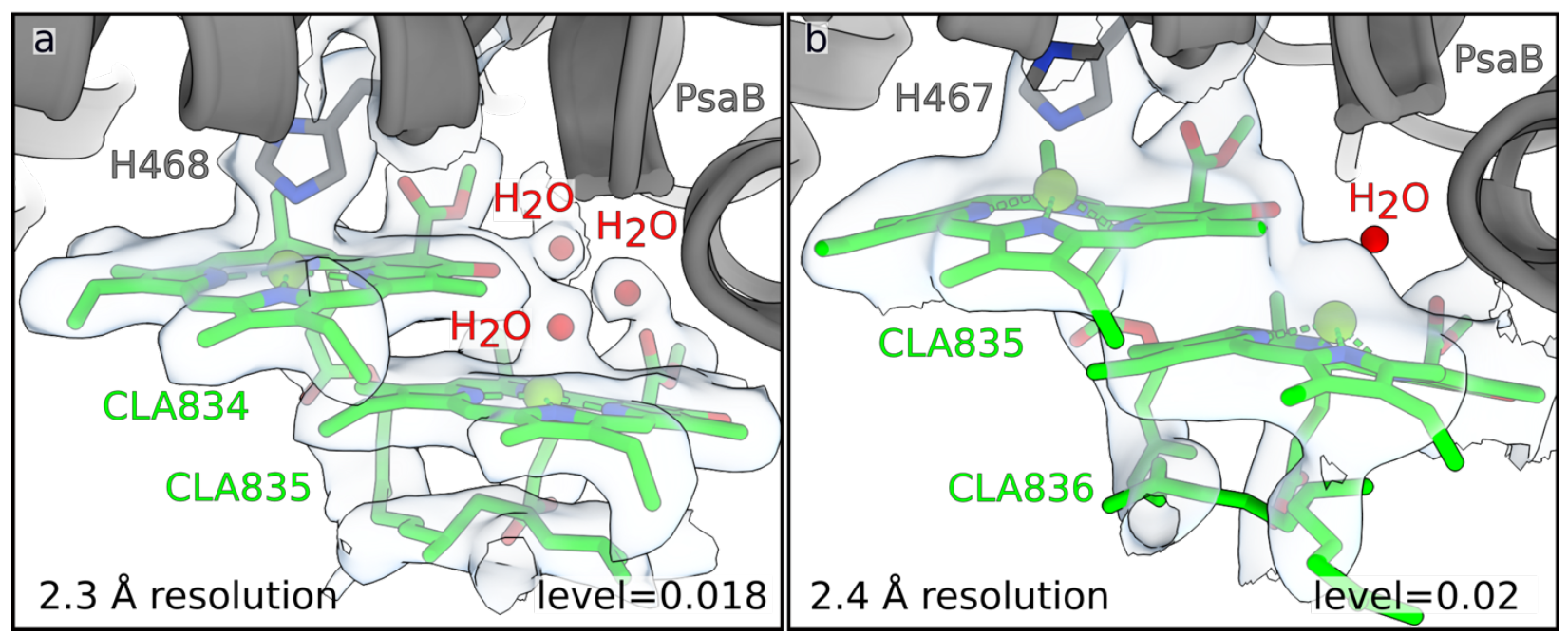

687 Extended Data Fig. 9: Comparison of the map/model quality with X-ray study. a, The 688 current density around CLA834-835 is shown in iso-surface representation at a local resolution of $6892.3 \AA$. Three water molecules involved in chlorophyll coordination are identified. Updated 690 libraries are used for correct chlorophyll modeling. b. The same region from X-ray study at $2.4 \AA$ 691 resolution ${ }^{17}$ lacks the density for waters, whereas the modeled water molecule has no density, and $\mathrm{Mg}$ atoms are not in the correct plane. 


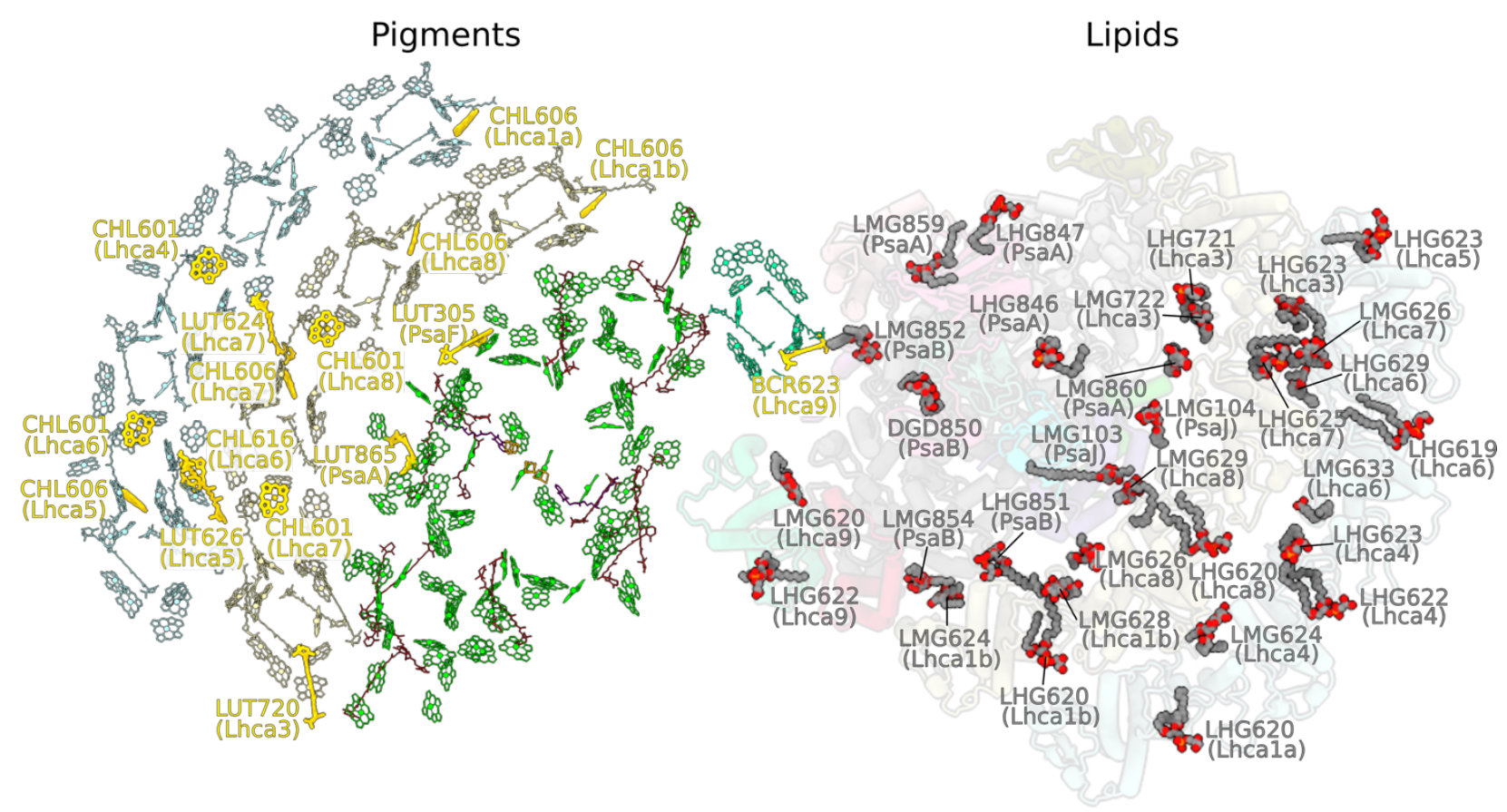

697

698 Extended Data Fig. 10: Overview of the pigments and lipids. Pigments (left) and lipids (right) 699 are shown from stroma. The newly identified pigments are highlighted in bold gold. 
Algae

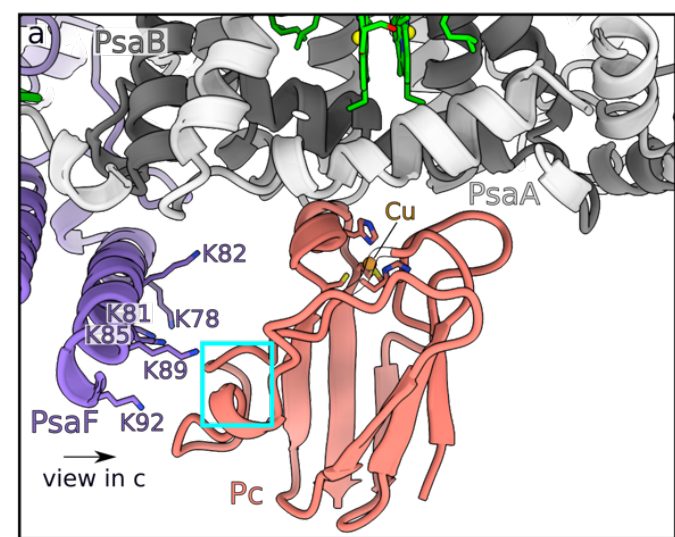

Plant (PDB ID: 6ZOO)

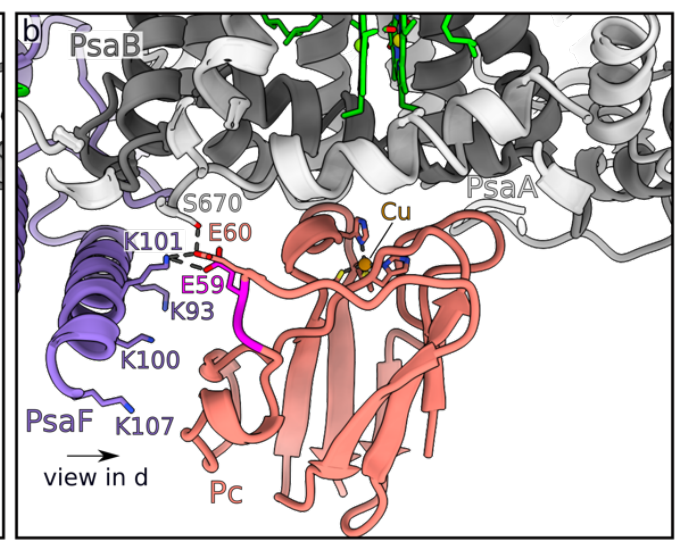

$\$ 90^{\circ}$

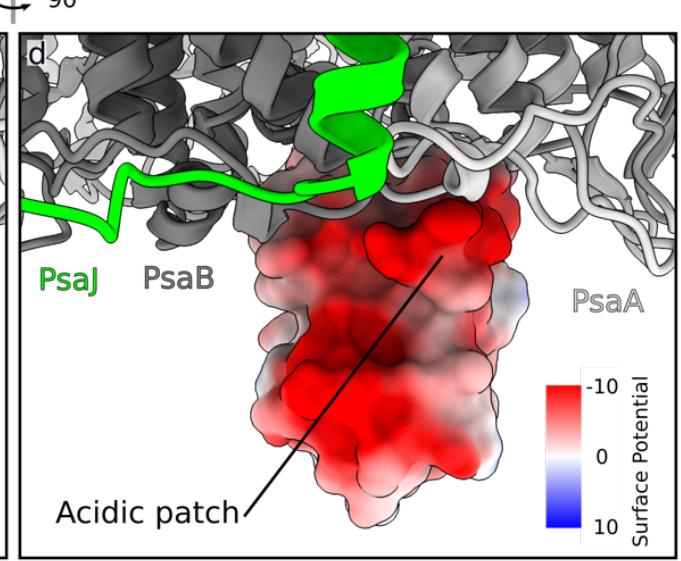

e

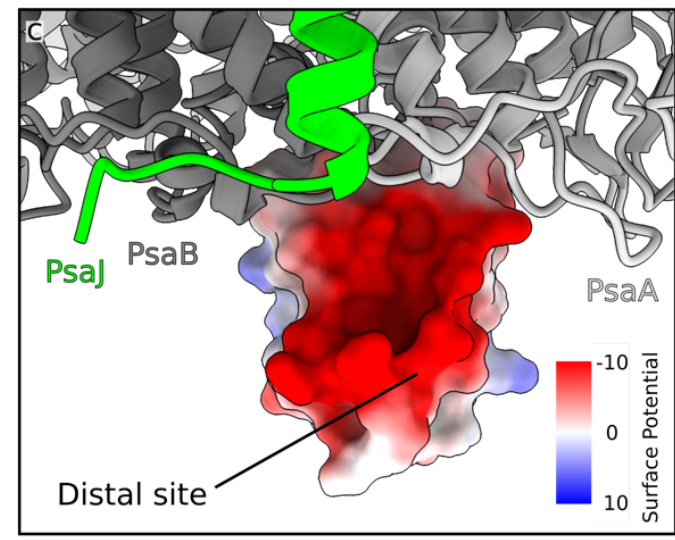

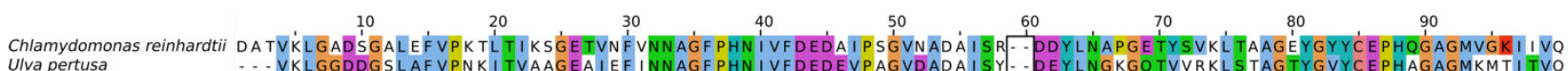

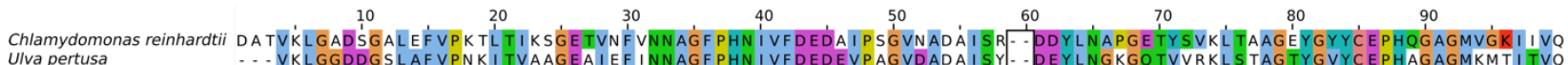
Ulva pertusa

Volvox carteri f. nagarier sis Chlorella sorokiniana

Mea mays

Oryza sativa

Hordeum vulgare

Capsicum annuum

Ricinus communis

Nicotiana benthamiana

Arabidopsis thaliana ..VKLGGDDGSLAFVPNKI TVAAGEAIEF INNAGFPHN IVFDEDEVPAGVDADA I SYY DEYLNGKGQTVVRKLSTAG TYGVYCEPHAGAGMKMTI TVQ

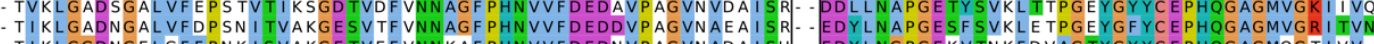

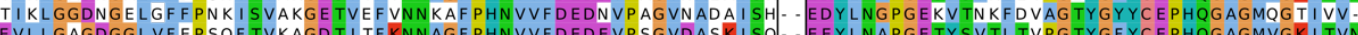
- EVLLGAGDGGLVFEPSQF TVKAGDTI TFKNNAGFPHNVVFDEDEVPSGVDAS IS

- EVLLGANGGVLVFEPNDF TVKSGE TI TFKNNAGFPHNVVFDEDAVPSGVDVS

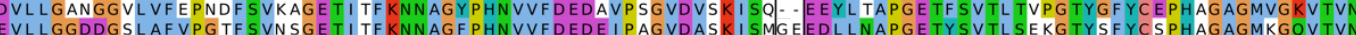
EILLGSDDGLLAFVPNSFSVAPGEK IVF KNNAGFPHN IVFDEDEVPSGVDAGK I SMSEEDLLNGPGE TYAVTL TEKG TYSFYCAPHQGAGMVG SVTVN VLLGSDDGLAFVPGNFSVSSGEKI TFKNNAGFPHNVVFDEDE I PAGVDVSKI SMPEEEYLNGPGE TYSVTLNEKG TYSFYCAPHQGAGMVGKVTVN

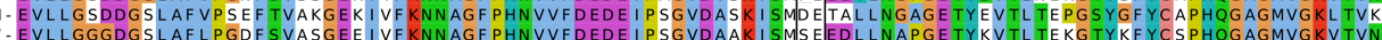

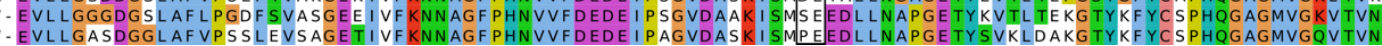

702 Extended Data Fig. 11: Plastocyanin binding site. a, Pc binding in C. reinhartdii (current

703 work). The positively charged residues of PsaF stabilize the interactions. The corresponding Pc

704 region that deviates from the plant counterpart is cyan. b, Pc binding in plants ${ }^{29}$. The two 705 inserted residues are magenta. c, $90^{\circ}$ rotated view with $\mathrm{Pc}$ surface shown with Coulomb potential 706 from the interface. d, the same view for a plant counterpart (PDB ID: 6ZOO), illustrating that the 707 acidic patch is shifted. e, Multiple sequence alignment of different species of the green lineage (algae and plants) showing that the inserted residues 58,59 occur in a subset of plants and do not represent a general case. 


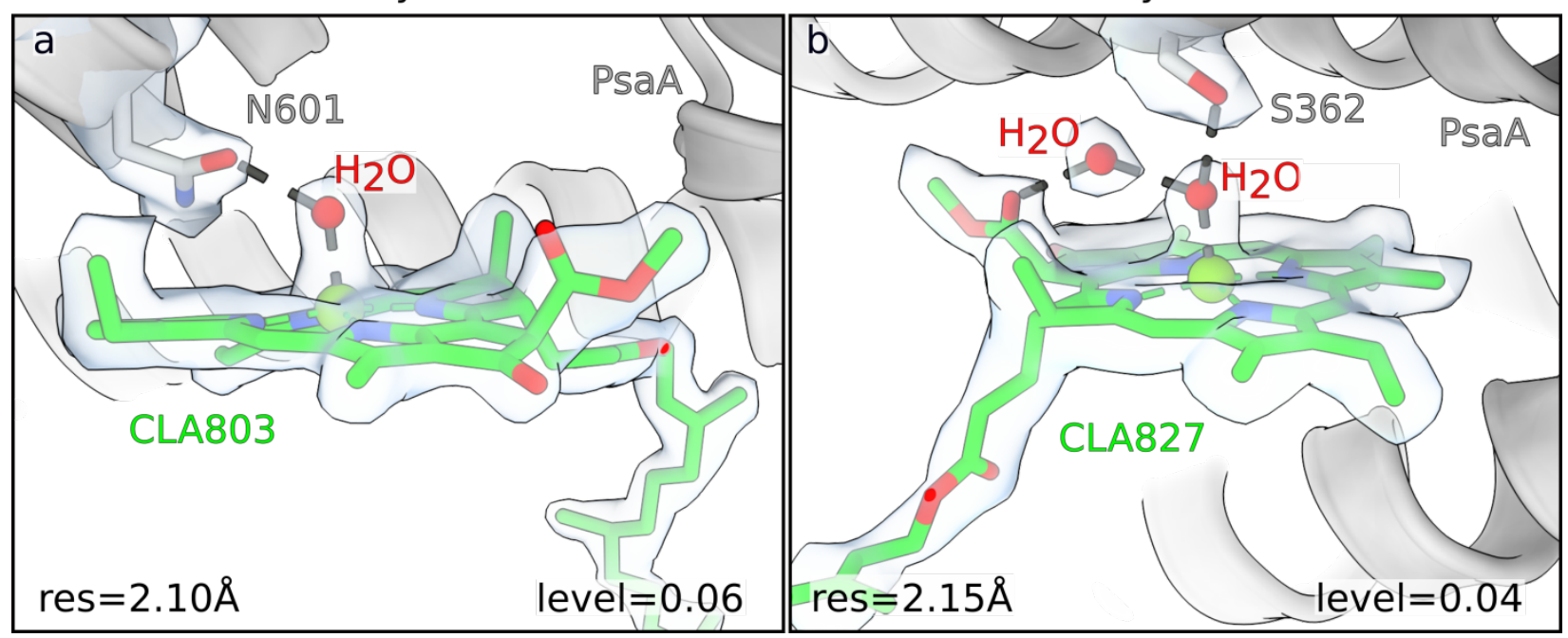

712 Extended Data Fig. 12: Mono- and di-hydrated chlorophylls. a, An example of a mono713 hydrated chlorophyll with the corresponding density. $\mathbf{b}$, An example of a di-hydrated chlorophyll 714 with the corresponding density. Local resolution and map levels are indicated. 
715 Supplementary Table 1: Protein composition of silver-stained spots from a 2D-PAGE of 716 solubilized $\boldsymbol{C}$. reinhardtii wild type thylakoids isolated from control and anoxic conditions.

\begin{tabular}{|c|c|}
\hline Spot & Identified proteins \\
\hline \multicolumn{2}{|c|}{ control } \\
\hline 1 & PsaA, PsaB \\
\hline 2 & $\beta$ mannosidase, $\mathrm{PsbB}, \mathrm{PsbC}$ \\
\hline 3 & Asa4, AtpB, PsbA, PsbB, PsbC \\
\hline 4 & Aaa1, AtpB, LhcbM3, PsbA, PsbB, PsbC \\
\hline 5 & $\beta$ mannosidase, Aaa1, ChlP, PsbB \\
\hline 6 & PsaH \\
\hline 7 & Lhcb5, LhcbM3, Nad5, PsbB, PsbC \\
\hline 8 & LhcbM3, PsbA, PsbB, PsbC \\
\hline 9 & LhcbM3, PsbB, PsbC \\
\hline 10 & Asa3, Asa4, Atp3 \\
\hline 11 & Lhcb4, Lhcb5, PetA, PsbA, PsbB, PsbC, PsbD \\
\hline 12 & Lhcb4, Lhcb5, LhcbM4, PsbA, PsbB, PsbD \\
\hline 13 & Lhcb4, Lhcb5, PetA, PsbA, PsbB, PsbD \\
\hline 14 & Lhcb4, Lhcb5, LhcbM3, LhcbM4, PsbA, PsbB, PsbD \\
\hline 15 & Lhcb4, Lhcb5, LhcbM3, PsbA, PsbB, PsbD, \\
\hline 16 & $\beta$ mannosidase, Lhca3, Lhca5, Lhca6, Lhca7, LhcbM1 \\
\hline 17 & Lhcb4, Lhcb5, LhcbM3, LhcbM4, PsbA, PsbD \\
\hline 18 & LhcbM1 \\
\hline 19 & LhcbM3, LhcbM4, LhcbM5, PsbA, PsbO \\
\hline 20 & Asa2, Lhca3, Lhca4, Lhca6, Lhcb5, LhcbM2, PsbB, PsbC \\
\hline \multicolumn{2}{|c|}{ anoxia } \\
\hline 1 & Atp2, PsaA, PsaB \\
\hline 2 & Asa2, PsbC \\
\hline 3 & Lhcb5, LhcbM3, PsbA, PsbD \\
\hline 4 & Lhcb4, Lhcb5, PsbA, PsbD \\
\hline 5 & LhcbM3, LhcbM4 \\
\hline 6 & LhcbM3, LhcbM4, PsaB, PsbO \\
\hline 7 & Lhca1, Lhca3, Lhca4, Lhca5, Lhca6, Lhca7, Lhca8 \\
\hline 8 & Lhca3, Lhca5, Lhca6, Lhca7, Lhca8, Lhcb5, LhcbM1 \\
\hline 9 & Lhca3, Lhca5, Lhca6, Lhca7, Lhca8 \\
\hline 10 & Lhca3, Lhca7, Lhca8 \\
\hline 11 & Lhca1, Lhca7, Lhca8, Lhca9, \\
\hline 12 & Lhca1, Lhca7, Lhca8, Lhca9, PsaD \\
\hline 13 & Asa7, Lhca1, Lhca9, Lhcb4, PsaD, PsbO \\
\hline 14 & PetC \\
\hline 15 & Asa7, Lhca1, PsaB, PsaD \\
\hline 16 & PsaF \\
\hline 17 & Nuo13, PsaF \\
\hline 18 & AtpG \\
\hline
\end{tabular}


bioRxiv preprint doi: https://doi.org/10.1101/2021.08.30.458224; this version posted August 31, 2021. The copyright holder for this preprint (which was not certified by peer review) is the author/funder, who has granted bioRxiv a license to display the preprint in perpetuity. It is made available under aCC-BY 4.0 International license.

\begin{tabular}{|l|l|}
19 & Nuo3, NuoP5 \\
\hline 20 & PsaH \\
\hline 21 & Asa6, PsaH \\
\hline 22 & PsaH \\
\hline 23 & PsaH \\
\hline
\end{tabular}

717

718 
719 Supplementary Table 2: Cryo-EM data collection, refinement and validation statistics of 720 PSI monomer and dimer of $C$. reinhartdii

$\begin{array}{llll}\begin{array}{l}\text { Dimer } \\ (\text { EMDB-xxxx })\end{array} & \begin{array}{l}\text { Symmetry expanded } \\ \text { dimer }\end{array} & \begin{array}{l}\text { Monomer } \\ (\text { EMDB-xxxx })\end{array} & \begin{array}{l}\text { Plastocyanin } \\ (\text { EMDB-xxxx) }\end{array} \\ (\text { PDB xxxx }) & \begin{array}{l}\text { (EMDB-xxxx) } \\ (\text { PDB xxxx) }\end{array} & \text { (PDB xxxx) } & (\text { PDB xxxx }) \\ & & & \end{array}$

\begin{tabular}{|c|c|c|c|c|}
\hline \multicolumn{5}{|l|}{$\begin{array}{l}\text { Data collection and } \\
\text { processing }\end{array}$} \\
\hline Magnification & 105,000 & 105,000 & 105,000 & $165,000 / 105,00$ \\
\hline Voltage (kV) & 300 & 300 & 300 & 300 \\
\hline Electron exposure $\left(\mathrm{e}-/ \AA^{2}\right)$ & 45.8 & 45.8 & 45.8 & $48 / 45.8$ \\
\hline Defocus range $(\mu \mathrm{m})$ & $-0.7-2.5$ & $-0.7-2.5$ & $-0.7-2.5$ & $-0.3,-1.1 /-0.7-2.5$ \\
\hline Pixel size $(\AA)$ & 0.84 & 0.84 & 0.84 & $0.51 / 0.84$ \\
\hline Initial particle images (no.) & 17,439 & 17,439 & 17,439 & $19,969 / 17,439$ \\
\hline Symmetry imposed & $\mathrm{C} 2$ & $\mathrm{C} 1$ & $\mathrm{C} 1$ & $\mathrm{C} 1$ \\
\hline Final particle images (no.) & 14,173 & 28,346 & 74,209 & 66,080 \\
\hline $\begin{array}{l}\text { Map resolution }(\AA) \\
\quad \text { FSC threshold } 0.143\end{array}$ & 2.97 & 2.74 & 2.31 & 2.55 \\
\hline \multicolumn{5}{|l|}{ Refinement } \\
\hline $\begin{array}{l}\text { Initial model used (PDB } \\
\text { code) }\end{array}$ & $6 \mathrm{JO5}$ & $6 \mathrm{JO5}$ & $6 \mathrm{JO5}$ & SWISS model \\
\hline $\begin{array}{l}\text { Map sharpening } B \text { factor } \\
\left(\AA^{2}\right)\end{array}$ & -28.80 & -21.00 & -23.05 & -21.05 \\
\hline \multicolumn{5}{|l|}{ Model composition } \\
\hline Non-hydrogen atoms & 100,830 & 56,460 & 50,370 & 729 \\
\hline Protein residues & 8,418 & 4,712 & 4,164 & 98 \\
\hline Ligands & 708 & 398 & 352 & 0 \\
\hline Waters & 160 & 88 & 515 & 0 \\
\hline \multicolumn{5}{|l|}{$\begin{array}{l}B \text { factors }\left(\AA^{2}\right) \\
(\min / \mathrm{max} / \text { mean })\end{array}$} \\
\hline Protein & $22.5 / 125.1 / 65.5$ & $48.7 / 154.0 / 80.3$ & 13.9/117.2/39.9 & $66.7 / 83.8 / 73.5$ \\
\hline Ligand & 25.5/118.9/66.9 & $51.0 / 148.5 / 79.45$ & $15.9 / 109.5 / 39.58$ & \\
\hline Waters & $25.4 / 115.3 / 64.0$ & $1.1 / 95.5 / 72.0$ & $13.4 / 57.5 / 32.1$ & \\
\hline \multicolumn{5}{|l|}{ R.m.s. deviations } \\
\hline Bond lengths (Å) & 0.009 & 0.009 & 0.009 & 0.002 \\
\hline Bond angles $\left(^{\circ}\right)$ & 1.065 & 1.069 & 1.085 & 0.494 \\
\hline \multicolumn{5}{|l|}{ Validation } \\
\hline MolProbity score & 1.16 & 1.16 & 1.15 & 1.20 \\
\hline Clashscore & 3.70 & 3.69 & 3.36 & 4.21 \\
\hline Poor rotamers $(\%)$ & 0.39 & 0.35 & 0.24 & 0.00 \\
\hline \multicolumn{5}{|l|}{ Ramachandran plot } \\
\hline Favored (\%) & 98.40 & 98.34 & 97.89 & 98.96 \\
\hline Allowed (\%) & 1.57 & 1.61 & 2.11 & 1.04 \\
\hline Disallowed (\%) & 0.02 & 0.04 & 0.00 & 0.00 \\
\hline
\end{tabular}


722 Supplementary Table 3: Chemical moieties involved in coordination of chlorophylls (per 723 monomer).

\begin{tabular}{|c|c|c|c|c|c|c|c|c|c|c|}
\hline \multicolumn{6}{|l|}{ Chlorophyll $a$} & \multicolumn{5}{|c|}{ Total: 199} \\
\hline & His & Met & $1 \times \mathrm{H}_{2} \mathrm{O}$ & $2 \mathrm{x} \mathrm{H}_{2} \mathrm{O}$ & Gln & $\begin{array}{l}\text { Back- } \\
\text { bone }\end{array}$ & Lipid & Asp & Glu & Asn \\
\hline All subunits & 84 & 2 & 13 & 22 & 13 & 11 & 10 & 5 & 28 & 11 \\
\hline PsaA & 32 & 1 & 3 & 4 & 3 & 1 & 1 & & & \\
\hline PsaB & 31 & 1 & 5 & & 1 & & 1 & 1 & & \\
\hline PsaF & & & & 2 & & & & 1 & & \\
\hline PsaG & 1 & & & & & & & 1 & & \\
\hline PsaJ & & & & & & & & & 1 & \\
\hline PsaK & 1 & & & 1 & & 1 & & 1 & & \\
\hline PsaL & 1 & & 1 & & & & & & & \\
\hline Lhca1_b & 2 & & & 3 & & 1 & 1 & & 3 & 1 \\
\hline Lhca1_a & 2 & & 1 & 2 & & 1 & 1 & & 3 & 1 \\
\hline Lhca3 & 2 & & 1 & 2 & 2 & 2 & & & 3 & 1 \\
\hline Lhca4 & 1 & & & 1 & 1 & & 1 & 1 & 3 & 2 \\
\hline Lhca5 & 3 & & & 2 & 1 & 2 & 1 & & 3 & 1 \\
\hline Lhca6 & 3 & & 1 & 1 & 1 & & 1 & & 3 & 1 \\
\hline Lhca7 & 2 & & 1 & 2 & 1 & 1 & 1 & & 3 & 1 \\
\hline Lhca8 & 2 & & & 2 & 1 & 1 & 1 & & 3 & 1 \\
\hline Lhca9 & 1 & & & & 2 & 1 & 1 & & 3 & 2 \\
\hline
\end{tabular}

724

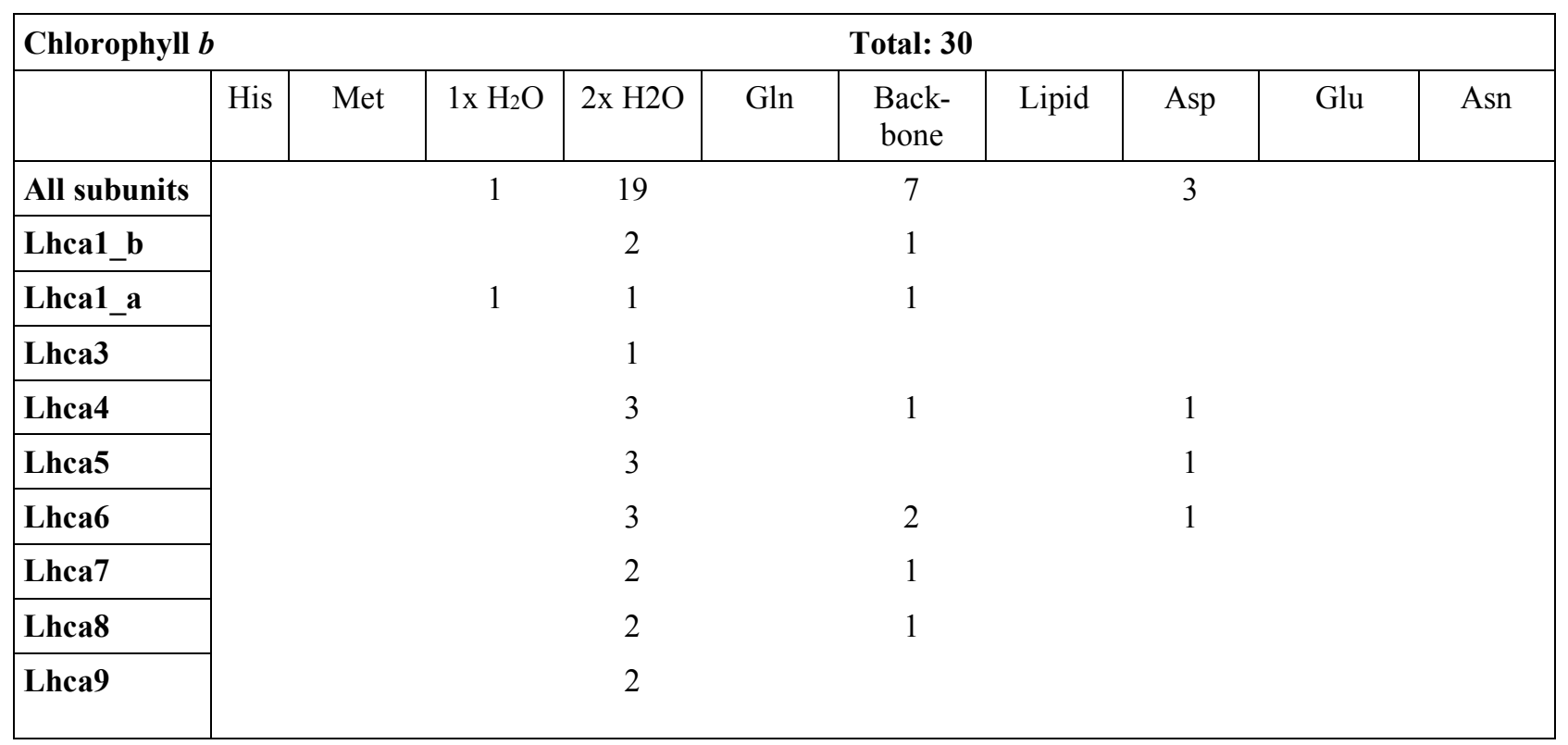

